\title{
Fermionic currents in topologically nontrivial braneworlds
}

\author{
S. Bellucci, ${ }^{1, *}$ A. A. Saharian, ${ }^{2, \dagger}$ D. H. Simonyan, ${ }^{2, *}$ and V. V. Vardanyan ${ }^{2,3,4,8}$ \\ ${ }^{1}$ INFN, Laboratori Nazionali di Frascati, Via Enrico Fermi 40, 00044 Frascati, Italy \\ ${ }^{2}$ Department of Physics, Yerevan State University, 1 Alex Manoogian Street, 0025 Yerevan, Armenia \\ ${ }^{3}$ Lorentz Institute for Theoretical Physics, Leiden University, 2333 CA Leiden, Netherlands \\ ${ }^{4}$ Leiden Observatory, Leiden University, 2300 RA Leiden, Netherlands
}

(Received 7 August 2018; published 29 October 2018)

\begin{abstract}
We investigate the influence of a brane on the vacuum expectation value (VEV) of the current density for a charged fermionic field in the background of locally AdS spacetime with an arbitrary number of toroidally compact dimensions and in the presence of a constant gauge field. Along compact dimensions, the field operator obeys quasiperiodicity conditions with arbitrary phases, and on the brane, it is constrained by the bag boundary condition. The brane is parallel to the AdS boundary, and it divides the space into two regions with different properties for the fermionic vacuum. In both these regions, the VEVs for the charge density and the components of the current density along noncompact dimensions vanish. The components along compact dimensions are decomposed into the brane-free and brane-induced contributions. The behavior of the latter in various asymptotic regions of the parameters is investigated. It particular, it is shown that the brane-induced contribution is mainly located near the brane and vanishes on the AdS boundary and on the horizon. An important feature is the finiteness of the current density on the brane. Applications are given to $Z_{2}$-symmetric braneworlds of the Randall-Sundrum type with compact dimensions for two classes of boundary conditions on the fermionic field. For the second one, we show that the contribution of the brane to the current does not vanish when the location of the brane tends to the AdS boundary. In odd spacetime dimensions, the fermionic fields realizing two inequivalent irreducible representations of the Clifford algebra and having equal phases in the periodicity conditions give the same contribution to the vacuum current density. Combining the contributions from these fields, the current density in odd-dimensional $C$-, $P$ - and $T$-symmetric models is obtained. In the special case of threedimensional spacetime, the corresponding results are applied for the investigation of the edge effects on the ground state current density induced in curved graphene tubes by an enclosed magnetic flux.
\end{abstract}

DOI: $10.1103 /$ PhysRevD.98.085020

\section{INTRODUCTION}

In a variety of quantum field-theoretical problems, the fields are defined on a manifold with a boundary and one must take care imposing suitable boundary conditions on the corresponding hypersurfaces. The boundaries may have different physical origins. Examples are interfaces between two media with different electromagnetic properties in condensed matter physics (e.g., media with different dielectric permittivities), various sorts of horizons in gravitational physics and in noninertial reference frames,

\footnotetext{
*bellucci@lnf.infn.it

†saharian@ysu.am

david.simonyan94@mail.ru

§ardanyan@lorentz.leidenuniv.nl
}

Published by the American Physical Society under the terms of the Creative Commons Attribution 4.0 International license. Further distribution of this work must maintain attribution to the author(s) and the published article's title, journal citation, and DOI. Funded by SCOAP . boundaries separating the spatial regions with different gravitational backgrounds (e.g., de Sitter bubbles in Minkowski spacetime), domain walls in the theory of phase transitions, and branes in higher-dimensional cosmologies and in string theories. In a number of physical problems, the model is formulated in nonglobally hyperbolic manifolds possessing a timelike boundary at spatial infinity. In order to preserve the information to be lost to, or gained from, spatial infinity, appropriate boundary conditions should be imposed. A well-known example of this kind is anti-de Sitter (AdS) spacetime [1]. Another class of conditions imposed on fields appear in models with compact spatial dimensions. The latter are an inherent feature of high-energy theories unifying physical interactions, like Kaluza-Klein and string theories. Depending on the periodicity conditions along compact dimensions different topologically inequivalent field configurations may arise [2]. The quantum effects arising from the nontrivial topological structure of the background spacetime include symmetry breaking, topological quantum phase transitions, instabilities in interacting field theories, 
and topological mass generation. The topological issues also play an important role in effective theories describing a number of condensed matter systems [3].

In the present paper, we consider the combined effects of background gravitational field and of two sorts of boundary conditions on the local properties of the vacuum state for a charged fermionic field. As the bulk geometry we take a locally AdS spacetime with an arbitrary number of toroidally compactified spatial dimensions (in Poincaré coordinates). The first kind of boundary condition is related to the presence of a brane parallel to the AdS boundary and the second one is related to the compactification of a part of spatial dimensions to a torus. We impose bag boundary condition on the brane and quasiperiodicity conditions with general phases along compact dimensions. The results are generalized for a boundary condition arising in $Z_{2}$ symmetric braneworld models of the Randall-Sundrum type with extra compact dimensions.

Our choice of AdS spacetime as a local bulk geometry has several motivations. First of all, AdS spacetime is maximally symmetric and a large number of problems in quantum field theory on curved backgrounds is exactly solvable. That is the case in the problem at hand. The corresponding investigations may help developing the research tools and insights to deal with less symmetric geometries. The AdS spacetime naturally appears as a ground state in extended supergravity and Kaluza-Klein theories and also as the near horizon geometry of the extremal black holes and domain walls. Moreover, the AdS spacetime has a constant negative curvature and the related length scale can serve as a regularization parameter for infrared divergences in interacting quantum field theories without reducing the number of symmetries [4]. The AdS geometry plays a crucial role in two exciting developments of the last two decades: the gauge/gravity duality and the braneworld scenario with large extra dimensions (for reviews see $[5,6])$. Braneworlds naturally appear in the string/M-theory context and provide an interesting alternative to address various problems in cosmological and particle physics. A number of particularly important implications of AdS geometry recently appeared in condensed matter physics (see, e.g., [7]).

Both types of constraints, induced by the presence of boundaries and by the compactification of spatial dimensions, give rise to the modification of the spectrum for vacuum fluctuations of quantum fields. As a result, the vacuum expectation values (VEVs) of physical quantities are shifted by an amount depending on the bulk and boundary geometries, and also on the boundary conditions imposed. This is the familiar Casimir effect (for reviews see [8]). The vacuum energy and the forces acting on the boundaries were among the main physical quantities of interest in the studies of this effect. In particular, motivated by the radion stabilization in braneworld models of the Randall-Sundrum type, the investigations of these quantities in the geometry of two parallel branes in AdS spacetime have attracted a great deal of attention (see, for instance, the references in $[9,10]$ ). In particular, the fermionic Casimir effect has been considered in [10-13] (for a recent discussion of the renormalised fermion expectation values on $\mathrm{AdS}$ spacetime in the absence of branes see, e.g., [14]). The vacuum energy, the Casimir forces and the VEV of the energy-momentum tensor in higher-dimensional generalizations of the AdS spacetime with compact internal spaces have been investigated in [15].

As another important local characteristic of the vacuum state for charged fields, bilinear in the field operator, here we consider the VEV of the current density for a fermionic field in background of locally AdS spacetime with compact dimensions in the presence of a brane. For a flat background geometry with an arbitrary number of toroidally compact dimensions, the zero and finite temperature expectation values of the charge and current densities for scalar and fermionic fields were investigated in Refs. [16-18]. The corresponding results for a special case of a three-dimensional spacetime with one and two compact spatial dimensions have been applied to the electronic subsystem of cylindrical and toroidal carbon nanotubes described within the framework of the effective Dirac model. The influence of additional boundaries on the vacuum charges and currents with applications to finite length carbon nanotubes is studied in $[19,20]$. This is the analog of the Casimir effect for the charge and current densities. The VEVs of the current densities for scalar and Dirac spinor fields in de Sitter and AdS spacetimes with toroidally compact subspace have been discussed in [21] and $[22,23]$, respectively, for scalar and fermionic fields. The effects of the branes in background of locally AdS bulk on the VEV of the current density for a scalar field with general curvature coupling parameter are investigated in $[24,25]$. The general case of the Robin boundary conditions on the branes was discussed and applications were given to Randall-Sundrum type braneworlds.

The organization of the present paper is as follows. In the next section, we describe the bulk geometry and the fields under consideration. The boundary and periodicity conditions are specified for a fermionic field. A complete set of positive and negative energy fermionic modes are described in Sec. III. By using the corresponding mode functions, in Sec. IV, we investigate the brane-induced effects on the current density along compact dimensions in the region between the brane and AdS boundary ( $\mathrm{L}$ region). The behavior of the current density in different asymptotic regions of the parameters is discussed in detail. Similar investigations for the region between the brane and AdS horizon (R region) are presented in Sec. V. In Sec. VI, the vacuum currents are considered in $Z_{2}$-symmetric braneworlds with a single brane and with an arbitrary number of toroidally compact spatial dimensions for two types of boundary conditions on the brane. The numerical results are given for the simplest generalization of the Randall-Sundrum 
model with a single extra compact dimension. The fermionic current density in parity and time-reversal symmetric models in odd-dimensional spacetimes is considered in Sec. VII. Applications are given to deformed carbon nanotubes described within the framework of the effective Dirac model in three-dimensional spacetime. The main results of the paper are summarized in Sec. VIII.

\section{BACKGROUND GEOMETRY AND THE FIELDS}

The background geometry we consider is described by the $(D+1)$-dimensional line element

$$
d s^{2}=e^{-2 y / a} \eta_{i k} d x^{i} d x^{k}-d y^{2},
$$

where $i, k=0,1, \ldots, D-1$ and $\eta_{i k}=\operatorname{diag}(1,-1, \ldots,-1)$ is the Minkowskian metric tensor in $D$-dimensional subspace with the coordinates $\left(x^{0}=t, x^{1}, \ldots, x^{D-1}\right)$. The local geometrical characteristics corresponding to (2.1) coincide with those for AdS spacetime with the curvature radius $a$. In particular, for the curvature scalar and the Ricci tensor one has $R=-D(D+1) / a^{2}$ and $R_{\mu \nu}=-D g_{\mu \nu} / a^{2}$. However, the global geometry we shall be concerned about is different. Namely, it will be assumed that the spatial dimension $x^{i}, i=p+1, \ldots, D-1$, is compactified to a circle with the length $L_{i}, 0 \leq x^{i} \leq L_{i}$. For the remaining coordinates, one has $-\infty<x^{i}<+\infty, i=1, \ldots, p$, and $-\infty<y<+\infty$. Hence, in the problem at hand the subspace $\left(x^{1}, \ldots, x^{D-1}\right)$ has the topology $R^{p} \times T^{q}$, $q=D-p-1$, where $T^{q}$ stands for a $q$-dimensional torus (for a discussion of physical effects in models with toroidal dimensions, see [26]). For further consideration, it is convenient, in addition to the coordinate $y$, to use the conformal coordinate $z$, defined as $z=a e^{y / a}$ with the range $0 \leq z<\infty$. In terms of the latter, the metric tensor is written in a conformally flat form

$$
g_{\mu \nu}=(a / z)^{2} \operatorname{diag}(1,-1, \ldots,-1),
$$

with the spacetime coordinates $x^{\mu}=\left(x^{0}, x^{1}, \ldots, x^{D-1}, x^{D}=z\right)$. The hypersurfaces $z=0$ and $z=\infty$ correspond to the AdS boundary and horizon, respectively. Note that for the proper length of the $i$ th compact dimension, measured by an observer with a fixed coordinate $z$, one has $L_{(p) i}=(a / z) L_{i}$.

We are interested in combined effects of the nontrivial topology and boundaries on the local characteristics of the vacuum state $|0\rangle$ for a massive fermionic field $\psi(x)$. Assuming the presence of an external Abelian gauge field $A_{\mu}(x)$, the corresponding field equation reads

$$
i \gamma^{\mu}\left(\partial_{\mu}+\Gamma_{\mu}+i e A_{\mu}\right) \psi(x)-m \psi(x)=0,
$$

where $e$ is the coupling between the fermionic and gauge fields and $\Gamma_{\mu}$ is the spin connection. For the curved spacetime Dirac matrices, one has $\gamma^{\mu}=e_{(b)}^{\mu} \gamma^{(b)}$, with $\gamma^{(b)}$ being the corresponding flat spacetime matrices and $e_{(b)}^{\mu}$ are the tetrad fields. For a fermionic field realizing the irreducible representation of the Clifford algebra, the matrices $\gamma^{(b)}$ are $N \times N$ matrices with $N=2^{[(D+1) / 2]}$, where the square brackets stand for the integer part of the enclosed expression. For odd $D$, the irreducible representation is unique up to a similarity transformation, whereas for even $D$ there are two inequivalent irreducible representations (see Sec. VII below). In the conformal coordinates $x^{\mu}$, with the metric tensor (2.2), we can take the tetrad fields in the form $e_{(b)}^{\mu}=(z / a) \delta_{b}^{\mu}$. The corresponding spin connection has the components $\Gamma_{k}=\eta_{k l} \gamma^{(D)} \gamma^{(l)} /(2 z)$ for $k=0, \ldots, D-1$, and $\Gamma_{D}=0$.

In the discussion below, we assume the presence of a boundary, parallel to the AdS boundary and located at $z=z_{0}$, on which the field operator is constrained by the bag boundary condition

$$
\left(1+i \gamma^{\mu} n_{\mu}\right) \psi(x)=0, \quad z=z_{0},
$$

where $n_{\mu}$ is the corresponding normal. The respective value of the $y$-coordinate we shall denote by $y_{0}, y_{0}=a \ln \left(z_{0} / a\right)$. Note that the physical distance from the boundary is given by $\left|y-y_{0}\right|$. Though the boundary under consideration my have different physical origins (e.g., in carbon nanotubes it corresponds to the edge of the tube), for the convenience of the discussion below we shall use the term "brane". It divides the background space into two regions: $0 \leq z \leq z_{0}$ and $z \geq z_{0}$. We shall refer to them as $\mathrm{L}$ and $\mathrm{R}$ regions (left and right regions), respectively. For the normal, one has $n_{\mu}=\delta_{\mu}^{D} a / z$ in the $\mathrm{L}$ region and $n_{\mu}=-\delta_{\mu}^{D} a / z$ in the $\mathrm{R}$ region. From (2.4), it follows that the normal component of the fermionic current vanishes on the brane. This feature is used in bag models of hadrons for confinement of quarks. Note that, though the geometrical characteristics of the background geometry do not depend on $z$, the boundary under consideration has a nonzero extrinsic curvature tensor with nonzero components $K_{i k}= \pm g_{i k} / a$, where the upper and lower signs correspond to the $\mathrm{L}$ and $\mathrm{R}$ regions, respectively. Related to this, the physical properties of the vacuum will be different in these regions.

The topology of the background space is nontrivial and, in addition to the boundary condition at $z=z_{0}$, we need to specify the periodicity conditions imposed on the field operator along compact dimensions. For the spatial dimension $x^{l}, l=p+1, \ldots, D-1$, we take the quasiperiodicity condition

$$
\psi\left(t, x^{1}, \ldots, x^{l}+L_{l}, \ldots, x^{D}\right)=e^{i \alpha_{l}} \psi\left(t, x^{1}, \ldots, x^{l}, \ldots, x^{D}\right),
$$

with a constant phase $\alpha_{l}$. The special cases of the most frequently used conditions with $\alpha_{l}=0$ and $\alpha_{l}=\pi$ correspond to untwisted and twisted fields. As for the gauge field, we assume the simplest configuration with $A_{\mu}=$ const. 
The corresponding effects on quantum properties of the vacuum are of the Aharonov-Bohm type and they are related to the nontrivial topology of the background space. The components of the vector potential along noncompact dimensions are simply removed by a gauge transformation and only the components along compact dimensions are physically relevant. Hence, our model is specified by the set of parameters $\left\{\alpha_{l}, A_{l}\right\}$ with $l=p+1, \ldots, D-1$.

Under the gauge transformation of the field variables $\psi(x)=\psi^{\prime}(x) e^{-i e \chi}, A_{\mu}=A_{\mu}^{\prime}+\partial_{\mu} \chi$, with the function $\chi=b_{\mu} x^{\mu}$, we obtain a new set of parameters $\left\{\alpha_{l}^{\prime}, A_{l}^{\prime}\right\}=$ $\left\{\alpha_{l}+e b_{l} L_{l}, A_{l}-b_{l}\right\}$. In particular, in the gauge with $b_{\mu}=$ $A_{\mu}$ the vector potential vanishes and for the new phases in the quasiperiodicity conditions for the field $\psi^{\prime}(x)$ one gets

$$
\tilde{\alpha}_{l}=\alpha_{l}+e A_{l} L_{l} .
$$

Hence, the effects of $\alpha_{l}$ and $A_{l}$ are not physically independent: the physical effects depend on these parameters in the form of the combination (2.6) which is invariant under the gauge transformation. In particular, a constant gauge field induces nontrivial effective phases for twisted and untwisted fields and vice versa: the nontrivial phases can be interpreted in terms of a constant gauge filed (or in terms of the magnetic flux). In what follows, we will work in the gauge $\left(\psi^{\prime}(x), A_{\mu}^{\prime}=0\right)$ omitting the primes. Along the $l$ th compact dimension the field $\psi^{\prime}(x)$ obeys the condition (2.5) with $\alpha_{l}$ replaced by $\tilde{\alpha}_{l}$ from (2.6). The part in the definition of the latter coming from the vector potential can be interpreted in terms of the magnetic flux $\Phi_{l}$ enclosed by the $l$ th dimension: $e A_{l} L_{l}=-2 \pi \Phi_{l} / \Phi_{0}$ (the minus sign comes from the fact that $A_{l}$ is the covariant component of the $(D+1)$-vector and it is related to the $l$ th component of the spatial vector $\mathbf{A}$ by $A_{l}=-\mathbf{A}_{l}$ ), with $\Phi_{0}=2 \pi / e$ being the flux quantum. Of course, this flux is fictive, it lives in the embedding space. However, it can be real flux if the model under consideration is realized as a brane in a higher dimensional spacetime. Another problem where the magnetic flux $\Phi_{l}$ has the real physical sense will be considered in Sec. VII.

\section{FERMIONIC MODES}

The VEVs of physical observables bilinear in the field operator are expressed in terms of the sums over a complete set of positive and negative energy fermionic modes $\left\{\psi_{\beta}^{(+)}, \psi_{\beta}^{(-)}\right\}$, where the set of quantum numbers $\beta$ specifies the solution. These modes obey the field equation (2.3) (with $A_{\mu}=0$ in the gauge under consideration), the boundary condition (2.4) and the quasiperiodicity conditions (2.5) with $\alpha_{l}$ replaced by $\tilde{\alpha}_{l}$. In order to find the solutions to the field equation, one needs to specify the representation of the flat spacetime Dirac matrices (for the construction of the Dirac matrices in an arbitrary number of spacetime dimensions, see, e.g., [27]). We find it convenient to use the representation (see also [23])

$$
\begin{array}{ll}
\gamma^{(0)}=\left(\begin{array}{cc}
0 & \chi_{0} \\
\chi_{0}^{\dagger} & 0
\end{array}\right), \quad \gamma^{(D)}=s i\left(\begin{array}{cc}
1 & 0 \\
0 & -1
\end{array}\right), \quad s= \pm 1 \\
\gamma^{(l)}=\left(\begin{array}{cc}
0 & \chi_{l} \\
-\chi_{l}^{\dagger} & 0
\end{array}\right), & l=1,2, \ldots, D-1,
\end{array}
$$

with $N / 2 \times N / 2$ matrices $\chi_{0}, \chi_{l}$. In even dimensional spacetimes, the irreducible representation is unique (up to a similarity transformation) and one can take $s=1$. In odddimensional spacetimes, the values $s=+1$ and $s=-1$ correspond to two inequivalent irreducible representations of the Clifford algebra. From the anticommutation relations for the Dirac matrices, we obtain the following relations

$$
\begin{aligned}
\chi_{l} \chi_{n}^{\dagger}+\chi_{n} \chi_{l}^{\dagger} & =2 \delta_{n l}, & & \chi_{l}^{\dagger} \chi_{n}+\chi_{n}^{\dagger} \chi_{l}=2 \delta_{n l}, \\
\chi_{0} \chi_{l}^{\dagger} & =\chi_{1} \chi_{0}^{\dagger}, & & \chi_{0}^{\dagger} \chi_{l}=\chi_{l}^{\dagger} \chi_{0}, \quad \chi_{0}^{\dagger} \chi_{0}=1,
\end{aligned}
$$

with $l, n=1,2, \ldots, D-1$. In the special case $D=2$, taking $\chi_{0}=\chi_{1}=1$, we get $\gamma^{(0)}=\sigma_{\mathrm{P} 1}, \gamma^{(1)}=i \sigma_{\mathrm{P} 2}, \gamma^{(2)}=s i \sigma_{\mathrm{P} 3}$, where $\sigma_{\mathrm{P} \mu}$ are the Pauli matrices.

With the flat spacetime matrices (3.1), substituting in the field equation (2.3) the Dirac matrices $\gamma^{\mu}=(z / a) \delta_{b}^{\mu} \gamma^{(b)}$, the complete set of the positive and negative energy solutions of the field equation can be found in a way similar to that we have described in Appendix of Ref. [23]. In accordance with the symmetry of the problem, the dependence of the mode functions on the coordinates $(t, \mathbf{x})=\left(t, x^{1}, \ldots, x^{D-1}\right)$ can be taken in the form of plane waves $e^{i \mathbf{k x}-i \omega t}, \mathbf{k} \mathbf{x}=\sum_{l=1}^{D-1} k_{i} x^{i}$, with the momentum $\mathbf{k}=$ $\left(k_{1}, \ldots, k_{D-1}\right)$ and the energy $\omega$. The mode functions are presented as

$\psi_{\beta}^{(+)}(x)=z^{\frac{D+1}{2}} e^{i \mathbf{k} \mathbf{x}-i \omega t}\left(\begin{array}{c}\frac{\mathbf{k} \chi \chi_{0}^{\dagger}+i \lambda-\omega}{\omega} Z_{m a+s / 2}(\lambda z) w^{(\sigma)} \\ i \chi_{0}^{\dagger} \frac{\mathbf{k} \chi \chi_{0}^{\dagger}+i \lambda+\omega}{\omega} Z_{m a-s / 2}(\lambda z) w^{(\sigma)}\end{array}\right)$,
$\psi_{\beta}^{(-)}(x)=z^{\frac{D+1}{2}} e^{i \mathbf{k} \mathbf{x}+i \omega t}\left(\begin{array}{c}i \chi_{0} \frac{\mathbf{k} \chi^{\dagger} \chi_{0}-i \lambda+\omega}{\omega} Z_{m a+s / 2}(\lambda z) w^{(\sigma)} \\ \frac{\mathbf{k} \chi^{\dagger} \chi_{0}-i \lambda-\omega}{\omega} Z_{m a-s / 2}(\lambda z) w^{(\sigma)}\end{array}\right)$,

where $w^{(\sigma)}, \sigma=1, \ldots, N / 2$, are one-column matrices having $N / 2$ rows and the elements $w_{l}^{(\sigma)}=\delta_{l \sigma}$. In (3.3), $\mathbf{k} \chi=\sum_{l=1}^{D-1} k_{l} \chi_{l}, 0 \leq \lambda<\infty, \omega=\sqrt{\lambda^{2}+k^{2}}, k=|\mathbf{k}|$ and

$$
Z_{\nu}(u)=c_{1} J_{\nu}(u)+c_{2} Y_{\nu}(u),
$$

is a linear combination of the Bessel and Neumann functions $J_{\nu}(u)$ and $Y_{\nu}(u)$. The coefficients $c_{1}$ and $c_{2}$ depend on the region under consideration and will be determined below separately in the $\mathrm{L}$ and $\mathrm{R}$ regions. 
For the components of the momentum along noncompact spatial dimensions, as usual, one has $-\infty<k_{i}<+\infty$, $i=1, \ldots, p$. The eigenvalues of the components along compact dimensions are quantized by the periodicity conditions:

$$
k_{l}=\frac{2 \pi n_{l}+\tilde{\alpha}_{l}}{L_{l}}, \quad n_{l}=0, \pm 1, \pm 2, \ldots,
$$

where $l=p+1, \ldots, D-1$. For $\tilde{\alpha}_{l}=2 \pi p_{l}$, with $p_{l}$ being an integer, the parameter $\tilde{\alpha}_{l}$ is removed from the problem by the redefinition of the quantum number $n_{l}$. Therefore, only the fractional part of $\tilde{\alpha}_{l} / 2 \pi$ is physically relevant. The set of quantum numbers $\beta$ specifying the modes is given by $\beta=\left(\lambda, \mathbf{k}_{(p)}, \mathbf{n}_{q}, \sigma\right)$, where $\mathbf{k}_{(p)}=\left(k_{1}, \ldots, k_{p}\right)$ is the momentum in the noncompact subspace and $\mathbf{n}_{q}=$ $\left(n_{p+1}, \ldots, n_{D-1}\right)$ determines the momentum in the compact subspace. The orthonormalization condition for the mode functions reads

$$
\int d^{D} x(a / z)^{D} \psi_{\beta}^{( \pm) \dagger} \psi_{\beta^{\prime}}^{( \pm)}=\delta_{\beta \beta^{\prime}},
$$

where $\delta_{\beta \beta^{\prime}}$ is understood as the Dirac delta function for the continuous components of $\beta$ and the Kronecker delta for discrete ones.

We are interested in the VEV of the current density $j^{\mu}=e \bar{\psi} \gamma \gamma^{\mu} \psi$, where for the Dirac conjugate one has $\bar{\psi}=\psi^{\dagger} \gamma^{(0)}$. Expanding the field operator in terms of the complete set of modes and using the anticommutation relations for the annihilation and creation operators, the $\operatorname{VEV}\left\langle 0\left|j^{\mu}\right| 0\right\rangle \equiv\left\langle j^{\mu}\right\rangle$ is presented in the form of the mode sum

$$
\left\langle j^{\mu}(x)\right\rangle=\frac{e}{2} \sum_{\beta}\left[\bar{\psi}_{\beta}^{(-)}(x) \gamma^{\mu} \psi_{\beta}^{(-)}(x)-\bar{\psi}_{\beta}^{(+)}(x) \gamma^{\mu} \psi_{\beta}^{(+)}(x)\right] .
$$

Here, $\sum_{\beta}$ stands for the integration over the continuous components of the collective index $\beta$ and for the summation over the discrete components. The functions $Z_{\nu}(u)$ in (3.3) and the eigenvalues for $\lambda$ are different in the $\mathrm{L}$ and $\mathrm{R}$ regions and we investigate the corresponding current densities separately.

\section{CURRENT DENSITY IN THE L REGION}

First we consider the region between the brane and the AdS boundary, corresponding to $0 \leq z \leq z_{0}$. In the range of the mass $m a \geq 1 / 2$ and for $c_{2} \neq 0$ in (3.4), the modes (3.3) are not normalizable. Hence, for this range, from the normalizability condition it follows that $c_{2}=0$ and the mode functions are given by (3.3) with $Z_{\nu}(u)=c_{1} J_{\nu}(u)$. From the boundary condition (2.4), it follows that the eigenvalues for the quantum number $\lambda$ are roots of the equation

$$
J_{m a-1 / 2}\left(\lambda z_{0}\right)=0,
$$

for both the cases $s= \pm 1$. We shall denote the corresponding positive roots with respect to $\lambda z_{0}$ by $\lambda_{n}=\lambda_{n}(m a)=$ $\lambda z_{0}, n=1,2, \ldots$, assuming that they are numerated in the ascending order, $\lambda_{n+1}>\lambda_{n}$. Note that the roots $\lambda_{n}$ do not depend on the location of the brane.

Now the mode functions are written as

$$
\begin{aligned}
& \psi_{\beta}^{(+)}(x)=C_{L \beta}^{(+)} z^{\frac{D+1}{2}} e^{i \mathbf{k} \mathbf{x}-i \omega t}\left(\begin{array}{c}
\frac{\mathbf{k} \chi \chi_{0}^{\dagger}+i \lambda-\omega}{\omega} J_{m a+s / 2}(\lambda z) w^{(\sigma)} \\
i \chi_{0}^{\dagger} \frac{\mathbf{k} \chi \chi_{0}^{\dagger}+i \lambda+\omega}{\omega} J_{m a-s / 2}(\lambda z) w^{(\sigma)}
\end{array}\right), \\
& \psi_{\beta}^{(-)}(x)=C_{L \beta}^{(-)} z^{\frac{D+1}{2}} e^{i \mathbf{k} \mathbf{x}+i \omega t}\left(\begin{array}{c}
i \chi_{0} \frac{\mathbf{k} \chi^{\dagger} \chi_{0}-i \lambda+\omega}{\omega} J_{m a+s / 2}(\lambda z) w^{(\sigma)} \\
\frac{\mathbf{k} \chi^{\dagger} \chi_{0}-i \lambda-\omega}{\omega} J_{m a-s / 2}(\lambda z) w^{(\sigma)}
\end{array}\right),
\end{aligned}
$$

where $\lambda=\lambda_{n} / z_{0}$. For a massless field, one has $\lambda_{n}=$ $\pi(n-1 / 2)$.

The normalization coefficients $C_{L \beta}^{( \pm)}$are determined from the condition (3.6), where the integration over $z$ is done in the region $\left[0, z_{0}\right]$ and on the right-hand side the Kronecker delta $\delta_{\lambda_{n} \lambda_{n^{\prime}}}=\delta_{n n^{\prime}}$ appears. By using the standard integral for the square of the Bessel function one finds

$$
\left|C_{\beta}^{( \pm)}\right|^{2}=\frac{J_{m a+1 / 2}^{-2}\left(\lambda_{n}\right)}{2(2 \pi)^{p} V_{q} a^{D} z_{0}^{2}},
$$

where $V_{q}=L_{p+1} \ldots L_{D-1}$ is the volume of the compact subspace. As seen, the normalization constants are the same for both the representations $s=1$ and $s=-1$.

For the range of masses $0 \leq m a<1 / 2$, the modes with $c_{2} \neq 0$ in (3.4) are normalizable. In this case, in order to determine the additional coefficient in the mode function one needs to specify a boundary condition for the field on the AdS boundary. This kind of boundary conditions for fermions have been discussed, e.g., in Refs. [28-30]. Here we shall consider a special type of boundary condition when the bag boundary condition is imposed on the hypersurface $z=z_{1}>0$ and then the limiting transition $z_{1} \rightarrow 0$ is taken. As it will be shown in the next section, this procedure leads to the mode functions which are given by (4.1) for all $m a \geq 0$.

We start our investigation for $\left\langle j^{\mu}\right\rangle$ with the charge density corresponding to the component $\mu=0$. Plugging the modes (4.1) in (3.7) we get

$$
\begin{aligned}
\left\langle j^{0}\right\rangle= & \frac{2 e}{a} z^{D+2} \sum_{\beta}\left|C_{\beta}^{( \pm)}\right|^{2} \frac{1}{\omega} w^{(\sigma) \dagger} \mathbf{k} \chi^{\dagger} \chi_{0} w^{(\sigma)} \\
& \times\left[J_{m a+s / 2}^{2}(\lambda z)-J_{m a-s / 2}^{2}(\lambda z)\right],
\end{aligned}
$$

where 


$$
\sum_{\beta}=\sum_{\mathbf{n}_{q}} \int d \mathbf{k}_{(p)} \sum_{n=1}^{\infty} \sum_{\sigma=1}^{N / 2}
$$

with $\mathbf{k}_{(p)}=\left(k_{1}, \ldots, k_{p}\right)$ being the momentum in the noncompact subspace. Now we note that for a $N / 2 \times N / 2$ matrix $M$ one has $\sum_{\sigma=1}^{N / 2} w^{(\sigma) \dagger} M w^{(\sigma)}=\operatorname{tr} M$. By taking into account the relations (3.2) it can be seen that $\operatorname{tr}\left(\chi_{l}^{+} \chi_{0}\right)=0$ and, hence, $\sum_{\sigma=1}^{N / 2} w^{(\sigma) \dagger} \mathbf{k} \chi^{\dagger} \chi_{0} w^{(\sigma)}=0$. From here, we conclude that the VEV of the charge density vanishes.

Now we turn to the $l$ th spatial component of the current density. By using the mode sum (3.7) with the modes (4.1), in a way similar to that for the charge density we can see that

$$
\begin{aligned}
\left\langle j^{l}\right\rangle= & -\frac{(2 \pi)^{-p} N e z^{D+2}}{2 V_{q} a^{D+1} z_{0}} \sum_{\mathbf{n}_{q}} \int d \mathbf{k}_{(p)} k_{l} \\
& \times \sum_{n=1}^{\infty} \frac{\sum_{j= \pm 1} J_{m a+j s / 2}^{2}\left(\lambda_{n} z / z_{0}\right)}{\sqrt{\lambda_{n}^{2}+z_{0}^{2} k^{2}} J_{m a+1 / 2}^{2}\left(\lambda_{n}\right)} .
\end{aligned}
$$

For $l=1, \ldots, p$, the integrand is an odd function with respect to the momentum $k_{l}$ and the corresponding component of the current density is zero, $\left\langle j^{l}\right\rangle=0$. Hence, a nonzero current density may appear along the compact dimensions only. This is a purely topological effect of the Aharonov-Bohm type and is induced by the nontrivial phases in the quasiperiodicity conditions (or, alternatively, by the enclosed magnetic fluxes). For $\tilde{\alpha}_{l}=2 \pi p_{l}$, with $p_{l}$ being an integer, after passing to the summation over $n_{l}^{\prime}=n_{l}+p_{l}$, we see that the contributions in (4.5) coming from the modes with positive and negative values of $k_{l}$ cancel each other and the resulting current density vanishes. Another important conclusion following from (4.5) is that the current densities for the representations with $s=1$ and $s=-1$ coincide. We will continue the investigation of the current density in the $\mathrm{L}$ region for the case $s=1$.

The representation (4.5) contains the eigenvalues $\lambda_{n}$ which are given implicitly, as the zeros of the Bessel function. In order to obtain a representation more convenient for the asymptotic and numerical analysis, and for explicit extraction of the brane-induced contribution, we apply to the series over $n$ a variant of the generalized Abel-Plana formula [31]

$$
\begin{aligned}
\sum_{n=1}^{\infty} \frac{f\left(\lambda_{n}\right)}{\lambda_{n} J_{m a+1 / 2}^{2}\left(\lambda_{n}\right)} & =\frac{1}{2} \int_{0}^{\infty} d u f(u)-\frac{1}{2 \pi} \int_{0}^{\infty} d u \frac{K_{m a-1 / 2}(u)}{I_{m a-1 / 2}(u)} \\
& \times\left[e^{(1 / 2-m a) \pi i} f(i u)+e^{(m a-1 / 2) \pi i} f(-i u)\right],
\end{aligned}
$$

valid for a function $f(u)$ analytic in the right half-plane of the complex variable $u$ (function $f(u)$ may have branch points on the imaginary axis, for the conditions imposed on this function see [31]). In (4.6), $I_{\nu}(u)$ and $K_{\nu}(u)$ are the modified Bessel functions. In the problem under consideration the function $f(u)$ is specified as

$f(u)=\frac{u}{\sqrt{u^{2}+z_{0}^{2} k^{2}}}\left[J_{m a+1 / 2}^{2}\left(u z / z_{0}\right)+J_{m a-1 / 2}^{2}\left(u z / z_{0}\right)\right]$

and has branch points $u= \pm i z_{0} k$.

After application of formula (4.6) to the series over $n$ in (4.5) and integration over the angular coordinates of the vector $\mathbf{k}_{(p)}$, the VEV of the current density is decomposed as

$$
\left\langle j^{l}\right\rangle=\left\langle j^{l}\right\rangle_{0}+\left\langle j^{l}\right\rangle_{b},
$$

where the term

$$
\begin{aligned}
\left\langle j^{l}\right\rangle_{0}= & -\frac{(4 \pi)^{-p / 2} N e z^{D+2}}{2 \Gamma(p / 2) V_{q} a^{D+1}} \sum_{\mathbf{n}_{q}} \int_{0}^{\infty} d k_{(p)} k_{(p)}^{p-1} k_{l} \\
& \times \int_{0}^{\infty} d \lambda \lambda \frac{J_{m a+s / 2}^{2}(\lambda z)+J_{m a-s / 2}^{2}(\lambda z)}{\sqrt{\lambda^{2}+k^{2}}}
\end{aligned}
$$

comes from the first integral in the right-hand side of (4.6) and coincides with the current density in the geometry without the brane (see [23]). The term

$$
\begin{aligned}
\left\langle j^{l}\right\rangle_{b}= & -\frac{4(4 \pi)^{-p / 2-1} N e z^{D+2}}{\Gamma(p / 2) V_{q} a^{D+1}} \sum_{\mathbf{n}_{q}} k_{l} \int_{0}^{\infty} d k_{(p)} k_{(p)}^{p-1} \int_{k}^{\infty} d u \\
& \times \frac{u}{\sqrt{u^{2}-k^{2}}} \frac{K_{m a-1 / 2}\left(u z_{0}\right)}{I_{m a-1 / 2}\left(u z_{0}\right)}\left[I_{m a+1 / 2}^{2}(u z)-I_{m a-1 / 2}^{2}(u z)\right],
\end{aligned}
$$

is the contribution induced by the brane. For a fixed $z$, the latter goes to zero in the limit $z_{0} \rightarrow \infty$.

The current density $\left\langle j^{l}\right\rangle_{0}$ in the brane-free geometry has been investigated in [23]. An alternative representation is given by

$$
\begin{aligned}
\left\langle j^{l}\right\rangle= & -\frac{e N a^{-D-1} L_{l}}{(2 \pi)^{(D+1) / 2}} \sum_{n_{l}=1}^{\infty} n_{l} \sin \left(\tilde{\alpha}_{l} n_{l}\right) \sum_{\mathbf{n}_{q-1}} \cos \left(\tilde{\boldsymbol{\alpha}}_{q-1} \cdot \mathbf{n}_{q-1}\right) \\
& \times \sum_{j=0,1} q_{m a-j}^{\frac{D+1}{2}}\left(1+\sum_{i=p+1}^{D-1} \frac{n_{i}^{2} L_{i}^{2}}{2 z^{2}}\right)
\end{aligned}
$$

where, $\mathbf{n}_{q-1}=\left(n_{p+1}, \ldots, n_{l-1}, n_{l+1}, \ldots, n_{D-1}\right), \tilde{\boldsymbol{\alpha}}_{q-1} \cdot \mathbf{n}_{q-1}=$ $\sum_{i=1, \neq l}^{D-1} \tilde{\alpha}_{i} n_{i}$. The function $q_{\nu}^{\mu}(x)$ is expressed in terms of the hypergeometric function $F(a, b ; c ; x)$ as

$$
\begin{aligned}
q_{\nu}^{\mu}(x)= & \frac{\sqrt{\pi} \Gamma(\nu+\mu+1)}{2^{\nu+1} \Gamma(\nu+3 / 2) x^{\nu+\mu+1}} \\
& \times F\left(\frac{\nu+\mu+1}{2}, \frac{\nu+\mu+2}{2} ; \nu+\frac{3}{2} ; \frac{1}{x^{2}}\right) .
\end{aligned}
$$


Note that for $\mu=1,2, \ldots$ (this corresponds to odd values of $D$ in (4.11)) one has $q_{\nu}^{\mu}(x)=(-1)^{\mu} \partial_{x}^{\mu} Q_{\nu}(x)$ with $Q_{\nu}(x)$ being the Legendre function of the second kind. For $\mu=$ $1 / 2,3 / 2, \ldots$ (even values of $D$ in (4.11)) the function $q_{\nu}^{\mu}(x)$ is expressed in terms of the elementary functions. In what follows, we will be mainly concerned about the braneinduced effects in the current density.

By taking into account that $k^{2}=k_{(p)}^{2}+k_{(q)}^{2}$, with

$$
k_{(q)}^{2}=\sum_{i=p+1}^{D-1}\left(2 \pi n_{i}+\tilde{\alpha}_{i}\right)^{2} / L_{i}^{2}
$$

the contribution (4.10) is further simplified by using the relation

$$
\begin{aligned}
& \int_{0}^{\infty} d k_{(p)} k_{(p)}^{p-1} \int_{k}^{\infty} d u \frac{u g(u)}{\sqrt{u^{2}-k^{2}}} \\
& \quad=\frac{\sqrt{\pi} \Gamma(p / 2)}{2 \Gamma((p+1) / 2)} \int_{k_{(q)}}^{\infty} d u u\left(u^{2}-k_{(q)}^{2}\right)^{\frac{p-1}{2}} g(u),
\end{aligned}
$$

for a given function $g(u)$. This leads to the following expression for the brane-induced contribution to the current density:

$$
\begin{aligned}
\left\langle j^{l}\right\rangle_{b}= & -\frac{N e A_{p} z^{D+2}}{V_{q} a^{D+1}} \sum_{\mathbf{n}_{q}} k_{l} \int_{k_{(q)}}^{\infty} d u u\left(u^{2}-k_{(q)}^{2}\right)^{\frac{p-1}{2}} \\
& \times \frac{K_{m a-1 / 2}\left(u z_{0}\right)}{I_{m a-1 / 2}\left(u z_{0}\right)}\left[I_{m a+1 / 2}^{2}(u z)-I_{m a-1 / 2}^{2}(u z)\right],
\end{aligned}
$$

with the notation

$$
A_{p}=\frac{(4 \pi)^{-(p+1) / 2}}{\Gamma((p+1) / 2)} .
$$

Note that the integrand in (4.15) is always negative. Both the brane-free and brane-induced contributions in the $l$ th component of the vacuum current density are odd periodic functions of the phase $\tilde{\alpha}_{l}$ and even periodic functions of $\tilde{\alpha}_{i}$ with $i \neq l$, with the period $2 \pi$. In particular, they are periodic functions of the magnetic flux with the period equal to the flux quantum $\Phi_{0}$. The charge flux through the hypersurface $x^{l}=$ const is given by $n_{l}^{(l)}\left\langle j^{l}\right\rangle$, where $n_{i}^{(l)}=$ $\delta_{i}^{l} a / z$ is the normal to that hypersurface. The product $a^{D} n_{l}^{(l)}\left\langle j^{l}\right\rangle$ depends on the variables having the dimension of length in the form of the dimensionless combinations $z_{0} / z, L_{i} / z, m a$. This feature is a consequence of the maximal symmetry of the AdS spacetime. Note that the ratio $L_{i} / z=L_{(p) i} / a$ is the proper length of the $i$ th compact dimension in units of the curvature radius $a$.

In order to further clarify the behavior of the current density, we pass to the investigation of the VEV (4.15) in special cases and in various asymptotic regions of the parameters. First we consider the current density of a massless fermionic field. In this case, the modified Bessel functions in (4.15) are expressed in terms of the elementary functions and one gets

$$
\begin{aligned}
\left\langle j^{l}\right\rangle_{b} & =\frac{2 N e A_{p} z^{D+1}}{V_{q} a^{D+1}} \sum_{\mathbf{n}_{q}} k_{l} \int_{k_{(q)}}^{\infty} d u \frac{\left(u^{2}-k_{(q)}^{2}\right)^{\frac{p-1}{2}}}{e^{2 u z_{0}}+1} \\
& =-\frac{2^{-3 p / 2} e N z^{D+1}}{\pi^{p / 2+1} V_{q} a^{D+1} z_{0}^{p}} \sum_{n=1}^{\infty} \frac{(-1)^{n}}{n^{p}} \sum_{\mathbf{n}_{q}} k_{l} g_{p / 2}\left(2 n z_{0} k_{(q)}\right),
\end{aligned}
$$

with the function

$$
g_{\nu}(x)=x^{\nu} K_{\nu}(x)
$$

The second representation in (4.17) is obtained from the first one by using the expansion $1 /\left(e^{x}+1\right)=$ $-\sum_{n=1}^{\infty}(-1)^{n} e^{-n x}$. The massless fermionic field is conformally invariant in an arbitrary number of spatial dimensions and the result (4.17) is obtained from the expression for the current density in the region between two boundaries at $z=0$ and $z=z_{0}$ on a locally Minkowskian bulk with compact dimensions $\left(x^{p+1}, \ldots, x^{D-1}\right)$ by using the conformal relation $\left\langle j^{l}\right\rangle_{b}=(z / a)^{D+1}\left\langle j^{l}\right\rangle_{b}^{(M)}$. Note that the boundary $z=0$ in the Minkowski bulk is the conformal image of the AdS boundary. We can see that $\left\langle j^{l}\right\rangle_{b}^{(M)}$ obtained from (4.17) coincides with the result from [17] (the sign difference is related to the fact that $\tilde{\alpha}_{i}$ in [17] corresponds to $-\tilde{\alpha}_{i}$ in the present paper).

The Minkowskian limit corresponds to $a \rightarrow \infty$ for fixed $y$ and $y_{0}$. In this limit, the conformal coordinates $z$ and $z_{0}$ are large, $z \approx a+y, z-z_{0} \approx y-y_{0}$, and, consequently, both the order and the argument of the modified Bessel functions in (4.15) are large. By using the corresponding uniform asymptotic expansions [32], to the leading order, we get

$$
\left\langle j^{l}\right\rangle_{b}^{(M)}=\frac{N e A_{p} m}{V_{q}} \sum_{\mathbf{n}_{q}} k_{l} \int_{m_{(q)}}^{\infty} d x\left(x^{2}-m_{(q)}^{2}\right)^{\frac{p-1}{2}} \frac{e^{-2 x\left|y-y_{0}\right|}}{x+m},
$$

with the notation $m_{(q)}=\sqrt{m^{2}+k_{(q)}^{2}}$. This expression coincides with the result from [19] for a boundary in a flat bulk with topology $R^{p+1} \times T^{q}$ (again, with the sign difference related to definition of the parameters $\tilde{\alpha}_{i}$ ). For a massless field, the current density induced by a single boundary in flat spacetime vanishes.

Now let us consider the behavior of the current density near the AdS boundary and near the brane for the fixed location of the brane. For points close to the AdS boundary, one has $z / z_{0} \ll 1$ and the main contribution to the integral in (4.15) comes from the region of the integration where the 
argument of the functions $I_{m a \pm 1 / 2}(u z)$ is small. By using the corresponding asymptotic expression, the leading-order term reads

$$
\begin{aligned}
\left\langle j^{l}\right\rangle_{b} \approx & \frac{N e A_{p} a^{-D-1} z^{D+2 m a+1}}{2^{2 m a-1} V_{q} \Gamma^{2}(m a+1 / 2)} \\
& \times \sum_{\mathbf{n}_{q}} k_{l} \int_{k_{(q)}}^{\infty} d u u^{2 m a}\left(u^{2}-k_{(q)}^{2}\right)^{\frac{p-1}{2}} \frac{K_{m a-1 / 2}\left(u z_{0}\right)}{I_{m a-1 / 2}\left(u z_{0}\right)},
\end{aligned}
$$

and on the AdS boundary the brane-induced contribution vanishes as $z^{D+2 m a+1}$. Note that the brane-free contribution behaves in a similar manner, $\left\langle j^{l}\right\rangle_{0} \propto z^{D+2 m a+1}$.

The representation (4.15) for the current density is not well suited for the investigation of the near-brane asymptotic. In order to obtain an alternative representation, we apply to the series over $n_{l}$ in the initial expression (4.5) the Abel-Plana-type formula [17]

$$
\begin{aligned}
\frac{2 \pi}{L_{l}} \sum_{n_{l}=-\infty}^{\infty} g\left(k_{l}\right) f\left(\left|k_{l}\right|\right)= & \int_{0}^{\infty} d u[g(u)+g(-u)] f(u) \\
& +i \int_{0}^{\infty} d u[f(i u)-f(-i u)] \\
& \times \sum_{j= \pm 1} \frac{g(i j u)}{e^{u L_{l}+i j \tilde{\alpha}_{l}}-1}
\end{aligned}
$$

for given functions $g(u), f(u)$ and with $k_{l}$ defined in (3.5) (formula (4.21) is reduced to the standard Abel-Plana formula in the special case $g(x)=1, \tilde{\alpha}_{l}=0$ ). For the series in (4.5), one has $g(u)=u$ and the first integral in (4.21) is zero. By making use of the relation

$$
\sum_{j= \pm 1} \frac{j}{e^{u L_{l}+i j \tilde{\alpha}_{l}}-1}=\frac{2}{i} \sum_{r=1}^{\infty} e^{-r u L_{l}} \sin \left(r \tilde{\alpha}_{l}\right),
$$

in the last term in (4.21), the integral over $u$ is expressed in terms of the modified Bessel function $K_{1}\left(n L_{l} \sqrt{\lambda^{2}+k^{2}-k_{l}^{2}}\right)$. Evaluating the remaining integral over $\mathbf{k}_{(p)}$ by using the formula from [33], the VEV of the current density is presented as (as it has been shown above, the current densities for the representations $s=1$ and $s=-1$ are the same and we consider the case $s=1$ )

$$
\begin{aligned}
\left\langle j^{l}\right\rangle= & -\frac{2 N e a^{-D-1} z^{D+2}}{(2 \pi)^{p / 2+1} V_{q} L_{l}^{p} z_{0}^{2}} \sum_{r=1}^{\infty} \frac{\sin \left(r \tilde{\alpha}_{l}\right)}{r^{p+1}} \sum_{\mathbf{n}_{q-1}} \sum_{n=1}^{\infty} \\
& \times g_{p / 2+1}\left(r L_{l} \sqrt{\lambda_{n}^{2} / z_{0}^{2}+k_{(q-1)}^{2}}\right) \\
& \times \frac{\sum_{j= \pm 1} J_{m a+j / 2}^{2}\left(\lambda_{n} z / z_{0}\right)}{J_{m a+1 / 2}^{2}\left(\lambda_{n}\right)},
\end{aligned}
$$

where the function $g_{\nu}(x)$ is defined by (4.18) and

$$
k_{(q-1)}^{2}=\sum_{i=p+1, \neq l}^{D-1}\left(2 \pi n_{i}+\tilde{\alpha}_{i}\right)^{2} / L_{i}^{2}
$$

Note that in the representation (4.23) the terms of the series over $n$ decay exponentially for large $\lambda_{n}$. In the case of a massless field, we have $\lambda_{n}=\pi(n-1 / 2)$ and the ratio of the Bessel functions in (4.23) is equal to $z_{0} / z$. In this case, we get the standard conformal relation with the corresponding representation of the current density between two boundaries in locally Minkowskian spacetime with compact dimensions.

The total current, per unit surface along the noncompact dimensions, is obtained by integration of (4.23):

$$
\begin{aligned}
V_{q} \int_{0}^{z_{0}} d z \sqrt{|g|}\left\langle j^{l}\right\rangle \\
=-\frac{2 N e}{(2 \pi)^{p / 2+1} L_{l}^{p}} \sum_{r=1}^{\infty} \frac{\sin \left(r \tilde{\alpha}_{l}\right)}{r^{p+1}} \\
\quad \times \sum_{\mathbf{n}_{q-1}} \sum_{n=1}^{\infty} g_{p / 2+1}\left(r L_{l} \sqrt{\lambda_{n}^{2} / z_{0}^{2}+k_{(q-1)}^{2}}\right) .
\end{aligned}
$$

Note that the dependence on the curvature radius of the background spacetime, on the mass of the field and on the location of the brane appears through the ratio $\lambda_{n} / z_{0}$. We recall that the roots $\lambda_{n}$ are completely determined by the parameter $m a$ and do not depend on the location of the brane.

In the model with a single compact dimension $x^{l}$ with the length $L_{l}(q=1, l=D-1)$, the formula (4.23) is specified to

$$
\begin{aligned}
\left.\left\langle j^{l}\right\rangle\right|_{q=1}= & -\frac{2 N e a^{-D-1} z^{D+2}}{(2 \pi)^{D / 2} L_{l}^{D-1} z_{0}^{2}} \sum_{r=1}^{\infty} \frac{\sin \left(r \tilde{\alpha}_{l}\right)}{r^{D-1}} \\
& \times \sum_{n=1}^{\infty} \frac{g_{D / 2}\left(r \lambda_{n} L_{l} / z_{0}\right)}{J_{m a+1 / 2}^{2}\left(\lambda_{n}\right)} \sum_{j= \pm 1} J_{m a+j / 2}^{2}\left(\lambda_{n} z / z_{0}\right) .
\end{aligned}
$$

An alternative expression in this special case is obtained from (4.15):

$$
\begin{aligned}
\left.\left\langle j^{l}\right\rangle_{b}\right|_{q=1}= & -N e \frac{A_{D-2} z^{D+2}}{a^{D+1} L_{l}} \sum_{n_{l}=-\infty}^{+\infty} k_{l} \int_{\left|k_{l}\right|}^{\infty} d u u\left(u^{2}-k_{l}^{2}\right)^{\frac{D-3}{2}} \\
& \times \frac{K_{m a-1 / 2}\left(u z_{0}\right)}{I_{m a-1 / 2}\left(u z_{0}\right)}\left[I_{m a+1 / 2}^{2}(u z)-I_{m a-1 / 2}^{2}(u z)\right] .
\end{aligned}
$$

In this and in the next sections, for numerical investigations of the current density, we consider the special case $D=4$ with a single compact dimension of the length $L_{l}=L$ and with the phase in the periodicity condition $\tilde{\alpha}_{l}=\tilde{\alpha}$. For this model, the corresponding formulas are obtained from (4.26) and (4.27) taking $p=2$ and $q=1$. 
In Fig. 1, we have plotted the dependence of the quantity $a^{D} n_{l}\left\langle j^{l}\right\rangle_{b} / e$, with $n_{l}=a / z$ (for the example at hand $l=3$ ), on the phase $\tilde{\alpha}$ and on the mass of the field (in units of the inverse curvature radius) for fixed values $z / z_{0}=0.95$ and $L / z_{0}=1 / 3$. Recall that $n_{l}\left\langle j^{l}\right\rangle_{b}$ is the charge flux through the spatial hypersurface $x^{l}=$ conts, induced by the brane. The current density is a periodic function of $\tilde{\alpha} / 2 \pi$ with the period 1 and we have plotted the dependence for the interval $-0.5 \leq \tilde{\alpha} / 2 \pi \leq 0.5$. Unlike the case of the $\mathrm{R}$ region (see below), the brane-induced current density in the $\mathrm{L}$ region does not vanish for a massless filed. With an initial increase of the mass the absolute value of the current density increases and after passing its maximum value tends to zero for large masses. In the latter limit, the orders of the modified Bessel functions in (4.15) are large and we can use the corresponding uniform asymptotic expansions. The contribution of the modes with $k_{(q)} \gg m$ and $k_{(q)}\left(z_{0}-z\right) \gg 1$ is suppressed by the factor $\exp \left[-2 k_{(q)}\left(z_{0}-z\right)\right]$. The dominant contribution comes from the modes with $k_{(q)}\left(z_{0}-z\right) \lesssim 1$ and the braneinduced VEV for large masses decays as $\left(z / z_{0}\right)^{2 m a}$, or as $\exp \left[-2 m a\left(y_{0}-y\right)\right]$ in terms of the proper distance from the brane.

An important conclusion made from the representation (4.23) is that the current density is finite on the brane and the corresponding value can be directly obtained putting $z=z_{0}$ :

$$
\begin{aligned}
\left\langle j^{l}\right\rangle_{z=z_{0}}= & -\frac{2 N e a^{-D-1} z_{0}^{D}}{(2 \pi)^{p / 2+1} V_{q} L_{l}^{p}} \sum_{r=1}^{\infty} \frac{\sin \left(r \tilde{\alpha}_{l}\right)}{r^{p+1}} \\
& \times \sum_{\mathbf{n}_{q-1}} \sum_{n=1}^{\infty} g_{p / 2+1}\left(r L_{l} \sqrt{\lambda_{n}^{2} / z_{0}^{2}+k_{(q-1)}^{2}}\right) .
\end{aligned}
$$

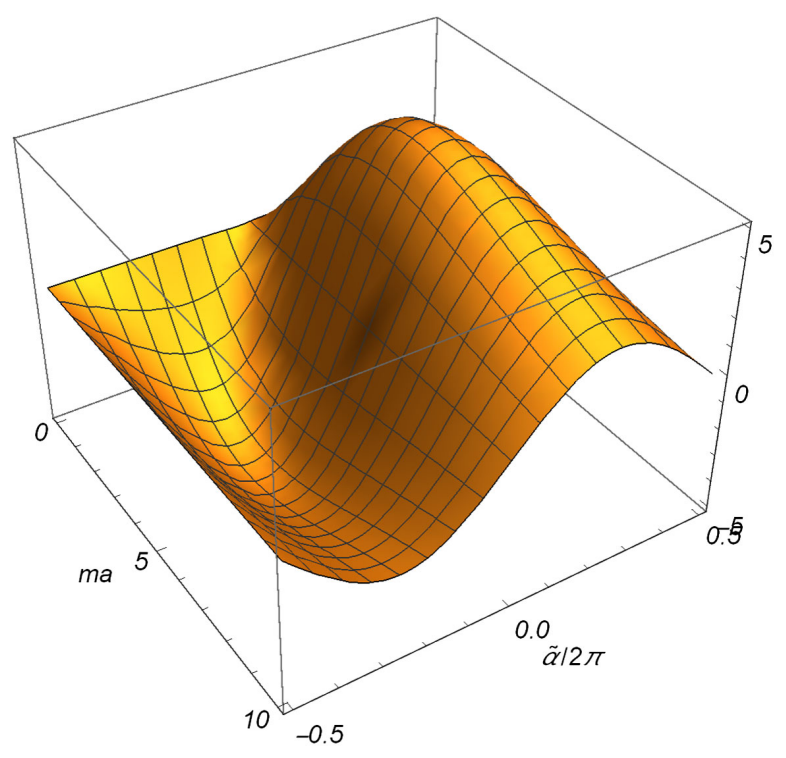

FIG. 1. The brane-induced charge flux along the compact dimension, $a^{D} n_{l}^{(l)}\left\langle j^{l}\right\rangle_{b}$, versus the mass and the phase in the periodicity condition for a $D=4$ model with a single compact dimension of the length $L$. The graph is plotted for $z / z_{0}=0.95$ and $L / z_{0}=1 / 3$.
In this sense, the behavior of the current density is essentially different from that for the VEVs of the fermion condensate and the energy-momentum tensor. The latter contain surface divergences well known from quantum field theory on manifolds with boundaries (see, for instance, [8]). The absence of the surface divergences in the VEV of the current density in the problem at hand can be understood as follows (see also a similar feature for scalar currents in $[24,25]$ ). The divergences in the VEVs of local observables are completely determined by the local geometrical characteristics of the bulk and boundary geometries. The compactification we have considered does not change these characteristics and they are the same as those for the problem in AdS spacetime without compact dimension. But in the latter problem the current density vanishes and, consequently contains no divergences. The toroidal compactification leaves the local geometry unchanged and, hence, no additional divergences will arise as a consequence of that. Comparing (4.28) with (4.25), we obtain the following relation between the current density on the brane and the current integrated over the coordinate $z$ :

$$
\int_{0}^{z_{0}} d z \sqrt{|g|}\left\langle j^{l}\right\rangle=\frac{a^{D+1}}{z_{0}^{D}}\left\langle j^{l}\right\rangle_{z=z_{0}} .
$$

Introducing in (4.15) a new integration variable $x=u z_{0}$, we see that the resulting integral is a function of two combinations $z_{0} k_{(q)}$ and $z / z_{0}$. For $z k_{(q)} \gg 1$, the arguments of the modified Bessel functions in the integrand are large. By using the corresponding asymptotics we find

$$
\left\langle j^{l}\right\rangle_{b} \approx \frac{N e A_{p} m z^{D}}{V_{q} a^{D}} \sum_{\mathbf{n}_{q}} k_{l} \int_{k_{(q)}}^{\infty} d u \frac{\left(u^{2}-k_{(q)}^{2}\right)^{\frac{p-1}{2}}}{u e^{2 u\left(z_{0}-z\right)}} .
$$

If, in addition, one has the condition $\left(z_{0}-z\right) k_{(q)} \gg 1$, the contribution of the corresponding modes to the braneinduced current density is suppressed by the factor $e^{-2 k_{(q)}\left(z_{0}-z\right)}$. For $z_{0}-z \gg L_{i}$, that condition is obeyed for all the modes and the integral in (4.30) is dominated by the contribution from the region near the lower limit and by the mode with the smallest value for $k_{(q)}$. Assuming that $\left|\tilde{\alpha}_{i}\right|<\pi$, this mode corresponds to $n_{i}=0$ for $i=p+1, \ldots$, $D-1$, and the leading-order term is presented as

$$
\left\langle j^{l}\right\rangle_{b} \approx \frac{N e m \tilde{\alpha}_{l} z^{D}\left(k_{(q)}^{(0)}\right)^{(p-3) / 2} e^{-2\left(z_{0}-z\right) k_{(q)}^{(0)}}}{2(4 \pi)^{(p+1) / 2} V_{q} L_{l} a^{D}\left(z_{0}-z\right)^{(p+1) / 2}},
$$

where

$$
k_{(q)}^{(0) 2}=\sum_{i=p+1}^{D-1} \tilde{\alpha}_{i}^{2} / L_{i}^{2} .
$$

From here, we conclude that the brane-induced contribution is mainly localized near the brane in the region $z_{0}-z \lesssim L_{i}$. Figure 2 displays the brane-induced current density as a 


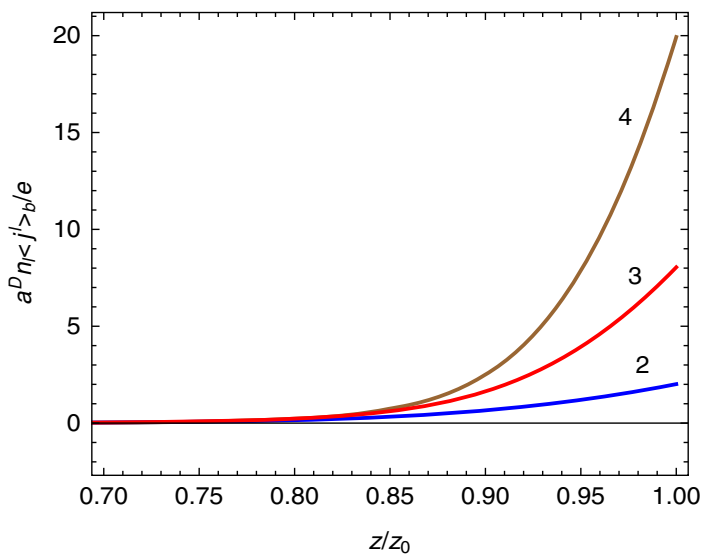

FIG. 2. The dependence of the brane-induced current density in the $L$ region on the ratio $z / z_{0}$. The graphs are plotted for different values of $z_{0} / L$ (numbers near the curves) and for fixed $m a=2$ and $\tilde{\alpha}=\pi / 2$.

function of $z / z_{0}$ for different values of the ratio $z_{0} / L$ (figures near the curves). The graphs are plotted for the same model as in Fig. 1 and for fixed values of $m a=2$ and $\tilde{\alpha}=\pi / 2$. Note that in terms of the proper distance from the brane one has $z / z_{0}=e^{-\left(y_{0}-y\right) / a}$. For the example presented, near the AdS boundary, $z \rightarrow 0$, the VEV decays as $\left(z / z_{0}\right)^{9}$.

Now let us consider the VEV of the current density as a function of the location of the brane. When the brane is close to the AdS horizon $\left(z_{0}\right.$ is large compared to the other length scales of the problem) the integral in (4.15) is dominated by the region where the argument $u z_{0}$ of the modified Bessel functions is large. By using the respective asymptotic formulas, we can see that the contribution of the mode with a given $\mathbf{n}_{q}$ is suppressed by the factor $e^{-2 k_{(q)} z_{0}}$. From here, it follows that the dominant contribution comes from the mode with the lowest possible value for $k_{(q)}$. Under the assumption $\left|\tilde{\alpha}_{i}\right|<\pi$, this mode corresponds to $n_{i}=0, \quad i=p+1, \ldots, D-1$, with $k_{(q)}=k_{(q)}^{(0)}$. To the leading order, one finds

$$
\begin{aligned}
\left\langle j^{l}\right\rangle_{b} \approx & -\frac{N e \tilde{\alpha}_{l} z^{D+2} k_{(q)}^{(0)(p+1) / 2} e^{-2 k_{(q)}^{(0)} z_{0}}}{2^{p+2} \pi^{(p-1) / 2} V_{q} L_{l} a^{D+1} z_{0}^{(p+1) / 2}} \\
& \times\left[I_{m a+1 / 2}^{2}\left(k_{(q)}^{(0)} z\right)-I_{m a-1 / 2}^{2}\left(k_{(q)}^{(0)} z\right)\right] .
\end{aligned}
$$

Hence, when the brane is close to the AdS horizon, the braneinduced current density is suppressed by the factor $e^{-2 k_{(q)}^{(0)} z_{0}}$.

If the brane is close to the AdS boundary, both $z_{0}$ and $z$ are small. In the investigation of the corresponding asymptotics, it is convenient to use the representation (4.23). The dominant contribution to the series over $\mathbf{n}_{q-1}$ comes from the terms with large values of $\left|n_{i}\right|$ with $i \neq l$ and we can replace the summation by the integration in accordance with

$$
\sum_{\mathbf{n}_{q-1}} f\left(k_{(q-1)}\right) \rightarrow 2 A_{q-2} \frac{V_{q}}{L_{l}} \int_{0}^{\infty} d x x^{q-2} f(x)
$$

where $k_{(q-1)}^{2}$ is defined by (4.24). After the integration over $x$ with the help of the formula

$$
\int_{0}^{\infty} d x x^{\beta-1} g_{\nu}\left(c \sqrt{x^{2}+b^{2}}\right)=\frac{2^{\beta / 2-1}}{c^{\beta}} \Gamma(\beta / 2) g_{\nu+\beta / 2}(b c),
$$

one gets $\left.\left\langle j^{l}\right\rangle \approx\left\langle j^{l}\right\rangle\right|_{q=1}$, where $\left.\left\langle j^{l}\right\rangle\right|_{q=1}$ is given by (4.26). In the limit under consideration, the argument of the function $g_{D / 2}\left(r \lambda_{n} L_{l} / z_{0}\right)$ is large and we use the corresponding asymptotic formula $g_{\nu}(x) \approx \sqrt{\pi / 2} x^{\nu-1 / 2} e^{-x}$. The dominant contribution comes from the lowest mode $r=1$ and we get

$$
\begin{aligned}
\left\langle j^{l}\right\rangle \approx & -\frac{N e z^{D+2} \sin \left(\tilde{\alpha}_{l}\right) \lambda_{1}^{(D-1) / 2} e^{-\lambda_{1} L_{l} / z_{0}}}{(2 \pi)^{(D-1) / 2} a^{D+1} L_{l}^{(D-1) / 2} z_{0}^{(D+3) / 2}} \\
& \times \frac{\sum_{j= \pm 1} J_{m a+j / 2}^{2}\left(\lambda_{1} z / z_{0}\right)}{J_{m a+1 / 2}^{2}\left(\lambda_{1}\right)},
\end{aligned}
$$

with the VEV suppressed by the factor $e^{-\lambda_{1} L_{l} / z_{0}}$.

For small values of $L_{l}$ compared with the lengths of the remaining compact dimensions, the dominant contribution to (4.15) comes from the modes with large values of $\left|n_{i}\right|$, $i \neq l$. In this case, to the leading order, we can replace the summation over $\mathbf{n}_{q-1}$ by the integration in accordance with (4.34). With this replacement, instead of $u$ we introduce a new integration variable $w$ as $u=\sqrt{x^{2}+w^{2}+k_{l}^{2}}$ and then polar coordinates in the plane $(x, w)$. After the integration over the angular variable, we get

$$
\left.\left\langle j^{l}\right\rangle_{b} \approx\left\langle j^{l}\right\rangle_{b}\right|_{q=1},
$$

with $\left.\left\langle j^{l}\right\rangle_{b}\right|_{q=1}$ from (4.27). The same relation takes place for the brane-free parts. If in addition $L_{l} \ll z_{0}$, the arguments of the modified Bessel functions in the integrand are large and we employ the corresponding asymptotic formulas. To the leading order, this gives

$$
\left\langle j^{l}\right\rangle_{b} \approx N e m \frac{A_{D-2} z^{D}}{a^{D} L_{l}} \sum_{n_{l}=-\infty}^{+\infty} k_{l}\left|k_{l}\right|^{D-3} \int_{1}^{\infty} d u \frac{\left(u^{2}-1\right)^{(D-3) / 2}}{u e^{2 u\left(z_{0}-z\right)\left|k_{l}\right|}} .
$$

For a massless field, this leading term vanishes. The expression on the right of (4.38) is further simplified under the condition $L_{l} \ll z_{0}-z$. In this case, the dominant contribution comes from the integration region near the lower limit and from the mode with the minimal value for 
$\left|k_{l}\right|$. Assuming $\left|\tilde{\alpha}_{l}\right|<\pi$, this mode corresponds to $n_{l}=0$ and we get

$\left\langle j^{l}\right\rangle_{b} \approx \frac{\operatorname{sgn}\left(\tilde{\alpha}_{l}\right) N e m\left|\tilde{\alpha}_{l}\right|^{(D-3) / 2}}{2^{D} \pi^{(D-1) / 2} a^{D} L_{l}^{(D-1) / 2}} \frac{z^{D} e^{-2\left(z_{0}-z\right) \mid \tilde{\alpha}_{l} / L_{l}}}{\left(z_{0}-z\right)^{(D-1) / 2}}$.

In this limit the sign of $\left\langle j^{l}\right\rangle_{b} / e$ coincides with that for $\tilde{\alpha}_{l}$.

In the opposite limit of large values $L_{l}$ it is convenient to use the representation (4.23). The argument of the function $g_{p / 2+1}(x)$ is large and we can use the corresponding asymptotic expression. The dominant contribution to the current density comes from the modes with $n=r=1$ and with the lowest value of $k_{(q-1)}$. Denoting the latter by $k_{(q-1)}^{(0)}$, to the leading order, one finds

$$
\begin{aligned}
\left\langle j^{l}\right\rangle= & -\frac{N e a^{-D-1} z^{D+2} \sin \left(\tilde{\alpha}_{l}\right) x^{(p+1) / 2} e^{-x}}{(2 \pi)^{(p+1) / 2} V_{q} L_{l}^{p} z_{0}^{2} J_{m a+1 / 2}^{2}\left(\lambda_{1}\right)} \\
& \times\left[J_{m a+1 / 2}^{2}\left(\lambda_{1} z / z_{0}\right)+J_{m a-1 / 2}^{2}\left(\lambda_{1} z / z_{0}\right)\right] .
\end{aligned}
$$

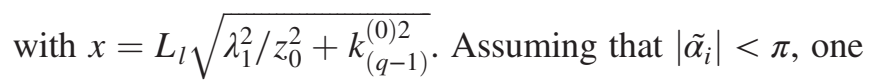
has

$$
k_{(q-1)}^{(0) 2}=\sum_{i=p+1, \neq l}^{D-1} \tilde{\alpha}_{i}^{2} / L_{i}^{2} .
$$

The nontrivial phases along compact dimensions $x^{i}, i \neq l$, enhance the suppression for the current density along the lth dimension. In Fig. 3, the current density is plotted as a function of $L / z_{0}$ for fixed values of $z / z_{0}=0.95$ (left panel) and $z / z_{0}=0.8$ (right panel). The graphs correspond to $m a=2$ and to the values of the phase $\tilde{\alpha}=2 \pi / 3$ (a), $\tilde{\alpha}=$ $\pi / 2$ (b), $\tilde{\alpha}=\pi / 3$ (c). The suppression for small values of $L$, described by (4.39), is seen in Fig. 3 .

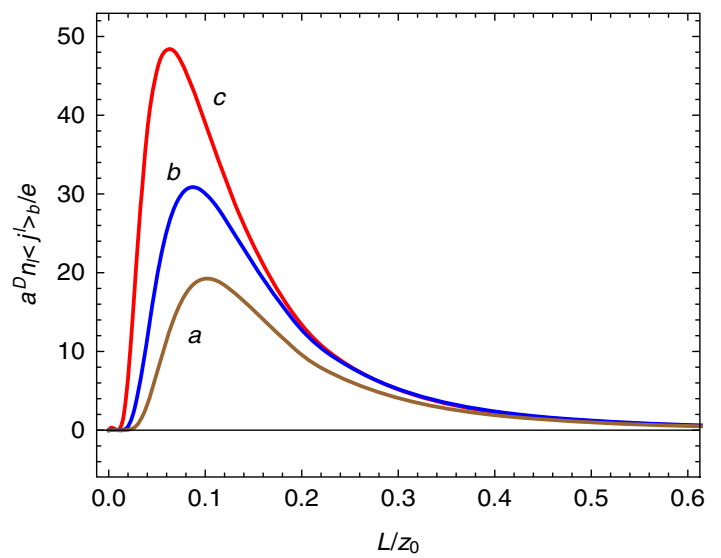

\section{CURRENTS IN THE R REGION}

In this section, we consider the current density in the region between the brane and the $\operatorname{AdS}$ horizon, $z_{0} \leq z<\infty$. The corresponding function $Z_{\nu}(u)$ is given by (3.4). From the boundary condition (2.4), it follows that $Z_{m a+1 / 2}\left(\lambda z_{0}\right)=0$. For the ratio of the coefficients in (3.4), this gives

$$
\frac{c_{2}}{c_{1}}=-\frac{J_{m a+1 / 2}\left(\lambda z_{0}\right)}{Y_{m a+1 / 2}\left(\lambda z_{0}\right)}
$$

and the function $Z_{\nu}(u)$ is expressed in terms of the function

$$
g_{\mu, \nu}(x, u)=J_{\mu}(x) Y_{\nu}(u)-J_{\nu}(u) Y_{\mu}(x) .
$$

In the $\mathrm{R}$ region, the spectrum for the quantum number $\lambda$ is continuous and the corresponding mode functions are written as

$$
\begin{aligned}
\psi_{\beta}^{(+)}(x)= & C_{R \beta}^{(+)} z^{\frac{D+1}{2}} e^{i \mathbf{k} \mathbf{x}-i \omega t} \\
& \times\left(\begin{array}{c}
\frac{\mathbf{k} \chi \chi_{0}^{\dagger}+i \lambda-\omega}{\omega} g_{m a+1 / 2, m a+s / 2}\left(\lambda z_{0}, \lambda z\right) w^{(\sigma)} \\
i \chi_{0}^{\dagger} \frac{\mathbf{k} \chi \chi_{0}^{\dagger}+i \lambda+\omega}{\omega} g_{m a+1 / 2, m a-s / 2}\left(\lambda z_{0}, \lambda z\right) w^{(\sigma)}
\end{array}\right), \\
\psi_{\beta}^{(-)}(x)= & C_{R \beta}^{(-)} z^{\frac{D+1}{2}} e^{i \mathbf{k} \mathbf{x}+i \omega t} \\
& \times\left(\begin{array}{c}
i \chi_{0} \frac{\mathbf{k} \chi^{\dagger} \chi_{0}-i \lambda+\omega}{\omega} g_{m a+1 / 2, m a+s / 2}\left(\lambda z_{0}, \lambda z\right) w^{(\sigma)} \\
\frac{\mathbf{k} \chi^{\dagger} \chi_{0}-i \lambda-\omega}{\omega} g_{m a+1 / 2, m a-s / 2}\left(\lambda z_{0}, \lambda z\right) w^{(\sigma)}
\end{array}\right) .
\end{aligned}
$$

The normalization constants are found from (3.6) with the integration over $z$ in the range $\left[z_{0}, \infty\right)$ and with $\delta\left(\lambda^{\prime}-\lambda\right)$ in the right-hand side:

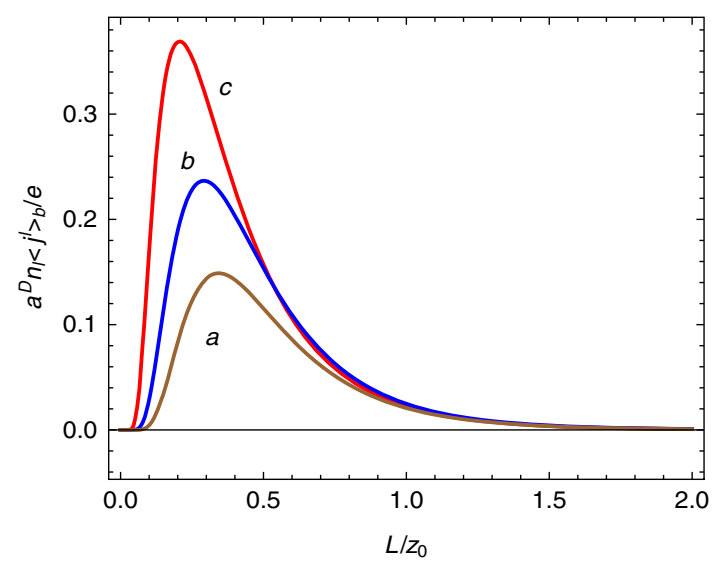

FIG. 3. The brane-induced charge flux along the compact dimension, $a^{D} n_{l}^{(l)}\left\langle j^{l}\right\rangle$, as a function of the rescaled length $L / z_{0}$ for separate values of the phase $\tilde{\alpha}=2 \pi / 3$ (a), $\tilde{\alpha}=\pi / 2$ (b), $\tilde{\alpha}=\pi / 3$ (c). For the mass, we have taken $m a=2$. For the left and right panels, $z / z_{0}=0.95$ and $z / z_{0}=0.8$, respectively. 


$$
\left|C_{R \beta}^{( \pm)}\right|^{2}=\lambda \frac{\left[J_{m a+1 / 2}^{2}\left(\lambda z_{0}\right)+Y_{m a+1 / 2}^{2}\left(\lambda z_{0}\right)\right]^{-1}}{4(2 \pi)^{p} V_{q} a^{D}} .
$$

Given the mode functions (5.3), the VEV of the current density is evaluated by using (3.7).

Let us consider the limiting transition $z_{0} \rightarrow 0$ for the mode functions (5.3). By taking into account that in this limit

$$
C_{R \beta}^{(+)} g_{m a+1 / 2, m a \pm 1 / 2}\left(\lambda z_{0}, \lambda z\right) \sim-\sqrt{\frac{\lambda}{4(2 \pi)^{p} V_{q} a^{D}}} J_{m a \pm 1 / 2}(\lambda z)
$$

(and the same with $C_{R \beta}^{(-)}$) we get the transition to the modes in boundary-free AdS spacetime that are analog of the modes (4.1) in the problem with the absence of the brane. Hence, we confirmed the statement in the previous section related to the boundary condition on the AdS boundary for the modes in the range of mass $0 \leq m a<1 / 2$.

Similarly to the case of the $\mathrm{L}$ region, we can see that the charge density and the components of the current density along noncompact dimensions vanish: $\left\langle j^{l}\right\rangle=0$ for $l=0,1, \ldots, p, D$. For the component along the $l$ th compact dimension, we get

$$
\begin{aligned}
& \left\langle j^{l}\right\rangle=-\frac{(4 \pi)^{-p / 2} N e z^{D+2}}{2 \Gamma(p / 2) V_{q} a^{D+1}} \sum_{\mathbf{n}_{q}} k_{l} \int_{0}^{\infty} d k_{(p)} k_{(p)}^{p-1} \\
& \times \int_{0}^{\infty} d \lambda \frac{\lambda}{\omega} \frac{\sum_{j= \pm 1} g_{m a+1 / 2, m a+j s / 2}^{2}\left(\lambda z_{0}, \lambda z\right)}{J_{m a+1 / 2}^{2}\left(\lambda z_{0}\right)+Y_{m a+1 / 2}^{2}\left(\lambda z_{0}\right)},
\end{aligned}
$$

with $l=p+1, \ldots, D-1$. From here, it follows that the current densities for the representations $s= \pm 1$ coincide in the $\mathrm{R}$ region. For the extraction of the brane-induced contribution, we use the identity

$\frac{g_{\nu, \mu}^{2}(x, y)}{J_{\nu}^{2}(x)+Y_{\nu}^{2}(x)}=J_{\mu}^{2}(y)-\frac{1}{2} \sum_{n=1,2} \frac{J_{\nu}(x)}{H_{\nu}^{(n)}(x)} H_{\mu}^{(n) 2}(y)$,

where $H_{\nu}^{(n)}(x), n=1,2$, are the Hankel functions. The contribution coming from the first term in the right-hand side of (5.7) gives the current density in the problem without the brane (see (4.9)) and the VEV is decomposed as in (4.8). In the brane-induced part, coming from the last term in (5.7), we rotate the integration contour over $\lambda$ by the angle $\pi / 2(-\pi / 2)$ for the term with $n=1 \quad(n=2)$. Introducing the modified Bessel functions and using the relation (4.14), for the brane-induced contribution to the $l$ th component of the current density we find

$$
\begin{aligned}
\left\langle j^{l}\right\rangle_{b}= & \frac{N e A_{p} z^{D+2}}{V_{q} a^{D+1}} \sum_{\mathbf{n}_{q}} k_{l} \int_{k_{(q)}}^{\infty} d u u\left(u^{2}-k_{(q)}^{2}\right)^{\frac{p-1}{2}} \\
& \times \frac{I_{m a+1 / 2}\left(u z_{0}\right)}{K_{m a+1 / 2}\left(u z_{0}\right)}\left[K_{m a+1 / 2}^{2}(u z)-K_{m a-1 / 2}^{2}(u z)\right] .
\end{aligned}
$$

This component is an odd periodic function of $\tilde{\alpha}_{l}$ and an even periodic function of $\tilde{\alpha}_{i}, i \neq l$, with the period $2 \pi$. The integrand in (5.8) is positive for $u>k_{(q)}$.

For a massless field, the brane-induced VEV (5.8) vanishes. This result could be directly obtained from the conformal relation of the problem under consideration in the case of massless field to the corresponding problem for a boundary in the flat spacetime bulk with compact dimensions. It is known that in the latter problem, for a massless fermionic field, the boundary-induced contribution vanishes (see [19]). The flat spacetime limit for a massive field can be obtained in a way similar to that we have demonstrated for the $\mathrm{L}$ region. The corresponding VEV is given by (4.19).

Figure 4 presents the brane-induced current density in the $\mathrm{R}$ region as a function of the mass and of the phase in the quasiperiodicity condition for fixed ratios $z_{0} / L=3$ and $z / z_{0}=1.15$. As for Fig. 1, we consider the model with $D=4$ and $(p, q)=(2,1)$ with the length of the compact dimension $L$ and the phase $\tilde{\alpha}$. With an initial increase of the mass the absolute value of the current density increases. After taking its maximal value the current density exponentially decays for large values of $m a$. For the modes with $k_{(q)}\left(z-z_{0}\right) \lesssim 1$, this decay is like $\left(z_{0} / z\right)^{2 m a}$.

With a new integration variable $x=u z_{0}$, the resulting integral in (5.8) depends on two dimensionless combinations

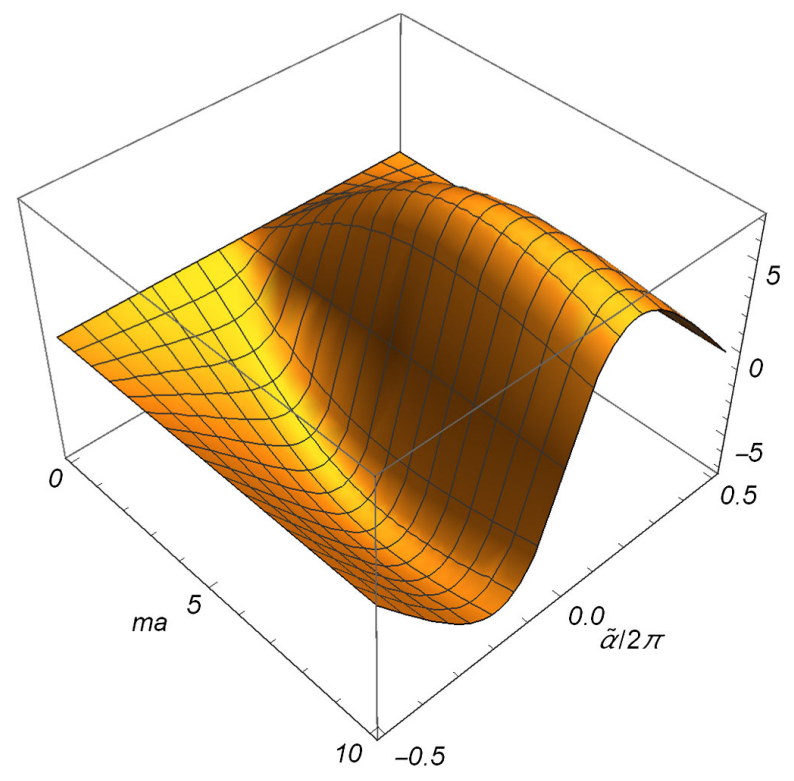

FIG. 4. The same as in Fig. 1 for $z / z_{0}=1.15$ ( $\mathrm{R}$ region). 
$z_{0} k_{(q)}$ and $z / z_{0}$. For $z_{0} k_{(q)} \gg 1$, by using the asymptotics for the modified Bessel functions for large arguments we can see that the corresponding leading term is obtained from the one in the $\mathrm{L}$ region, given by (4.30), by the replacement $z_{0}-z \rightarrow z-z_{0}$. If additionally one has the condition $\left(z-z_{0}\right) k_{(q)} \gg 1$, the contribution of the mode with a given $k_{(q)}$ decays as $e^{-2 k_{(q)}\left(z-z_{0}\right)}$. In particular, for $z-z_{0} \gg L_{i}$ that condition is valid for all the modes and for the leading term we get the expression that is obtained from (4.31) making the replacement $z_{0}-z \rightarrow z-z_{0}$. Hence, the leading term is the same for the $\mathrm{L}$ and $\mathrm{R}$ regions. It is mainly localized near the brane in the region $\left|z_{0}-z\right| \lesssim L_{i}$.

For the investigation of the behavior of the current density in some asymptotic regions of the parameters, we find it convenient to provide another representation. It is obtained from (5.6) by using the formula (4.21) for the summation of the series over $n_{l}$. By calculations similar to those for the $\mathrm{L}$ region, one gets

$$
\begin{aligned}
\left\langle j^{l}\right\rangle= & -\frac{N e a^{-D-1} z^{D+2}}{(2 \pi)^{p / 2+1} V_{q} L_{l}^{p}} \sum_{r=1}^{\infty} \frac{\sin \left(r \tilde{\alpha}_{l}\right)}{r^{p+1}} \sum_{\mathbf{n}_{q-1}} \int_{0}^{\infty} d \lambda \lambda \\
& \times g_{p / 2+1}\left(r L_{l} \sqrt{\lambda^{2}+k_{(q-1)}^{2}}\right) \\
& \times \frac{\sum_{j= \pm 1} g_{m a+1 / 2, m a+j / 2}^{2}\left(\lambda z_{0}, \lambda z\right)}{J_{m a+1 / 2}^{2}\left(\lambda z_{0}\right)+Y_{m a+1 / 2}^{2}\left(\lambda z_{0}\right)},
\end{aligned}
$$

with the function $g_{\nu}(x)$ from (4.18). In particular, from this representation it follows that the current density is finite on the brane with the value

$$
\begin{aligned}
\left\langle j^{l}\right\rangle_{z=z_{0}}= & -\frac{16 N e a^{-D-1} z_{0}^{D}}{(2 \pi)^{p / 2+3} V_{q} L_{l}^{p}} \sum_{r=1}^{\infty} \frac{\sin \left(r \tilde{\alpha}_{l}\right)}{r^{p+1}} \sum_{\mathbf{n}_{q-1}} \int_{0}^{\infty} \frac{d u}{u} \\
& \times \frac{g_{p / 2+1}\left(r L_{l} \sqrt{u^{2} / z_{0}^{2}+k_{(q-1)}^{2}}\right)}{J_{m a+1 / 2}^{2}(u)+Y_{m a+1 / 2}^{2}(u)} .
\end{aligned}
$$

For a massless field, by using the expressions for the functions $J_{ \pm 1 / 2}(x)$ and $Y_{ \pm 1 / 2}(x)$, from (5.9) it can be seen that

$$
\begin{aligned}
\left.\left\langle j^{l}\right\rangle\right|_{m=0}= & -\frac{2 N e(z / a)^{D+1}}{(2 \pi)^{(p+3) / 2} V_{q} L_{l}^{p+1}} \sum_{r=1}^{\infty} \frac{\sin \left(r \tilde{\alpha}_{l}\right)}{r^{p+2}} \\
& \times \sum_{\mathbf{n}_{q-1}} g_{(p+3) / 2}\left(r L_{l} k_{(q-1)}\right) .
\end{aligned}
$$

This result is conformally related to the current density in flat spacetime with toroidally compact dimensions in the absence of boundaries, obtained in [17] (with the sign difference related to different definitions of $\tilde{\alpha}_{i}$; note that in [17] the number of noncompact dimensions is $p$ instead of $p+1$ as in the present paper). This again shows that, for a massless field, the brane-induced contribution in the R-region vanishes.
Another representation for the current density in the R-region is obtained from (5.9) by using the identity (5.7) and rotating the integration contours for the terms with $n=1,2$ in a way similar to that for (5.8). This leads to the formula

$$
\begin{aligned}
\left\langle j^{l}\right\rangle= & \left\langle j^{l}\right\rangle_{0}+\frac{N e a^{-D-1} z^{D+2}}{(2 \pi)^{p / 2+1} V_{q} L_{l}^{p}} \sum_{r=1}^{\infty} \frac{\sin \left(r \tilde{\alpha}_{l}\right)}{r^{p+1}} \\
& \times \sum_{\mathbf{n}_{q-1}} \int_{k_{(q-1)}}^{\infty} d \lambda \lambda w_{p / 2+1}\left(r L_{l} \sqrt{\lambda^{2}-k_{(q-1)}^{2}}\right) \\
& \times \frac{I_{m a+1 / 2}\left(\lambda z_{0}\right)}{K_{m a+1 / 2}\left(\lambda z_{0}\right)}\left[K_{m a+1 / 2}^{2}(\lambda z)-K_{m a-1 / 2}^{2}(\lambda z)\right],
\end{aligned}
$$

with the function

$$
w_{\nu}(x)=x^{\nu} J_{\nu}(x) .
$$

The second term in the right-hand side of (5.12) is the contribution induced by the brane $\left(\left\langle j^{l}\right\rangle_{b}\right.$ in the notation used before).

For a fixed location of the brane and at distances from it larger than the curvature radius, one has $y-y_{0} \gg a$. In terms of the conformal coordinate, this corresponds to $z \gg z_{0}$. If additionally we assume that $z \gg L_{i}$, the integral in (5.8) is dominated by the contribution from the region near the lower limit of the integration and from the mode with $n_{i}=0$ (under the assumption $\left.\left|\tilde{\alpha}_{i}\right|<\pi\right), i=p+1, \ldots, D-1$. By using the asymptotic expression of the Macdonald function for large arguments, the leading-order contribution to the braneinduced part of the current density is presented as

$$
\begin{aligned}
\left\langle j^{l}\right\rangle_{b} \approx & \frac{N e z^{D-(p+1) / 2} m \tilde{\alpha}_{l}}{2^{p+2} \pi^{(p-1) / 2} V_{q} L_{l} a^{D}} \frac{I_{m a+1 / 2}\left(z_{0} k_{(q)}^{(0)}\right)}{K_{m a+1 / 2}\left(z_{0} k_{(q)}^{(0)}\right)} \\
& \times k_{(q)}^{(0)(p-3) / 2} e^{-2 z k_{(q)}^{(0)}} .
\end{aligned}
$$

Note that the large values for $z$ correspond to points near the AdS horizon. As it has been shown in [23], in that region the effects of the gravitational field on the brane-free part of the current density are small and one has the simple relation $\left\langle j^{l}\right\rangle_{0} \approx(z / a)^{D+1}\left\langle j^{l}\right\rangle_{0}^{(\mathrm{M})}$ with the current density in flat spacetime with toroidal spatial dimensions. This shows that near the AdS horizon the contribution $\left\langle j^{l}\right\rangle_{0}$ dominates the VEV. In Fig. 5, the brane-induced current density in the R region is depicted as a function of $z / z_{0}$ for separate values of the ratio $z_{0} / L$ (numbers near the curves) and for fixed values $m a=2, \tilde{\alpha}=\pi / 2$. As seen from the graphs in Fig. 5, for fixed values of $z_{0}$ and $z$, near the brane the current density increases with decreasing $L\left(z_{0} / L\right.$ increases $)$. At large distances from the brane the situation is opposite: with decreasing $L$ the current density decreases. This is also seen 


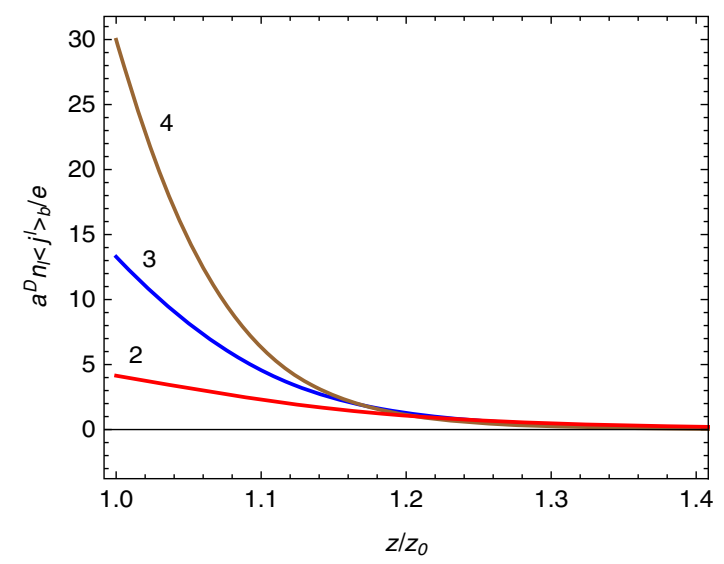

FIG. 5. The same as in Fig. 2 for the R region.

from the asymptotic estimate (5.14) with $z k_{(q)}^{(0)}=z|\tilde{\alpha}| / L$. From the analysis presented above, it follows that at large distances from the brane the VEV decays as $e^{\left.-2\left(z-z_{0}\right)\right) \tilde{\alpha} \mid / L}$ and the sign of $\left\langle j^{l}\right\rangle_{b} / e$ coincides with that for $\tilde{\alpha}$.

Let us consider the VEV of the current density as a function of the location of the brane. In the limit when the brane is close to the AdS boundary, $z_{0}$ is small and in (5.8) we replace the cylindrical functions with the arguments $u z_{0}$ by their asymptotics for small arguments. The leadingorder term is given by

$$
\begin{aligned}
\left\langle j^{l}\right\rangle_{b} \approx & \frac{2^{1-2 m a} N e A_{p} a^{-D-1} z^{D+2} z_{0}^{2 m a+1}}{V_{q}(2 m a+1) \Gamma^{2}(m a+1 / 2)} \\
& \times \sum_{\mathbf{n}_{q}} k_{l} k_{(q)}^{p+2 m a+2} \int_{1}^{\infty} d x x^{2 m a+2} \\
& \times\left(x^{2}-1\right)^{\frac{p-1}{2}}\left[K_{m a+1 / 2}^{2}\left(x z k_{(q)}\right)-K_{m a-1 / 2}^{2}\left(x z k_{(q)}\right)\right] .
\end{aligned}
$$

In this limit, for a fixed $z$, the brane-induced contribution decays as $z_{0}^{2 m a+1}$. For the location of the brane close to the AdS horizon, $z_{0}$ is large. The limiting case $z_{0} k_{(q)} \gg 1$ has

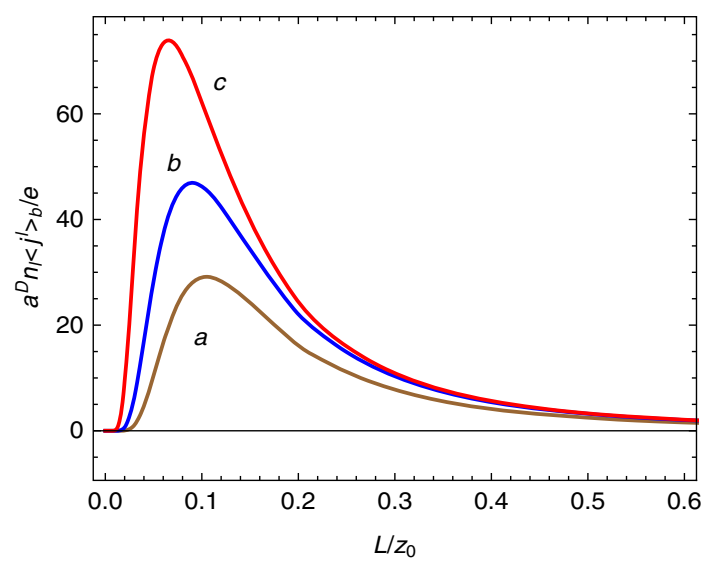

been already discussed above. The corresponding asymptotic is given by (4.30) with the replacement $z_{0}-z \rightarrow z-z_{0}$.

The asymptotic behavior of the current density, as a function of the length $L_{l}$, is investigated in a way similar to that for the L region. If $L_{l}$ is much smaller than the lengths of the remaining compact dimensions, the leading-order term coincides with the current density in the model with a single compact dimension $x^{l}$, when the remaining dimensions are decompactified. If additionally $L_{l} \ll z$, this term is transformed to the form that is obtained from (4.38) with the replacement $z_{0}-z \rightarrow z-z_{0}$. With the same replacement in (4.39), the asymptotic formula is obtained under the condition $L_{l} \ll z-z_{0}$. Hence, for small values of $L_{l}$ we have an exponential suppression by the factor $e^{-2\left(z-z_{0}\right)\left|\tilde{\alpha}_{l}\right| / L_{l}}$.

For large values of $L_{l}$, it is convenient to use the representation (5.9). The argument of the function $g_{p / 2+1}(x)$ is large and we use the corresponding asymptotic expression. Two cases should be considered separately. For $k_{(q-1)}^{(0)} \neq 0$ (at least one of the phases $\tilde{\alpha}_{i},\left|\tilde{\alpha}_{i}\right|<\pi, i \neq l$, is nonzero), the integral in (5.9) is dominated by the contribution from the region near the lower limit and by the mode with $r=1, k_{(q-1)}=k_{(q-1)}^{(0)}$. Using the asymptotics for the Bessel and Neumann functions for small arguments, the leadingorder term is expressed as

$\left\langle j^{l}\right\rangle \approx-\frac{N e a^{-D-1} z^{D+1+2 m a} \sin \left(\tilde{\alpha}_{l}\right) k_{(q-1)}^{(0)(p / 2+m a+1)}}{2^{m a+1 / 2}(2 \pi)^{(p+1) / 2} \Gamma(m a+1 / 2) V_{q} L_{l}^{p / 2+m a}} e^{-L_{l} k_{(q-1)}^{(0)}}$,

with an exponential suppression as a function of $L_{l}$. For $\tilde{\alpha}_{i}=0, i \neq l$, we have $k_{(q-1)}^{(0)}=0$ and, again, the leading contribution comes from the mode with $n_{i}=0, i \neq l$. Using the asymptotic expressions for the Bessel and Neumann functions, the integral with the function $g_{p / 2+1}\left(r L_{l} \lambda\right)$ in the integrand is expressed in terms of the gamma function and we get

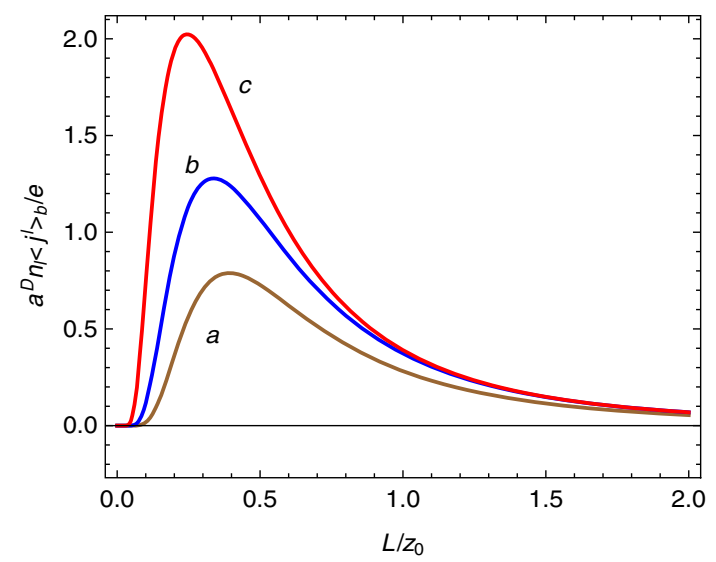

FIG. 6. The same as in Fig. 3 in the R region for $z / z_{0}=1.05$ (left panel) and $z / z_{0}=1.2$ (right panel). 


$$
\begin{aligned}
\left\langle j^{l}\right\rangle \approx & -\frac{e N a^{-D-1} z^{D+2 m a+1} \Gamma(m a+(p+3) / 2)}{\pi^{p / 2+1} V_{q} L_{l}^{p+2 m a+1} \Gamma(m a+1 / 2)} \\
& \times \sum_{r=1}^{\infty} \frac{\sin \left(r \tilde{\alpha}_{l}\right)}{r^{p+2 m a+2}},
\end{aligned}
$$

with a power-law decay as a function of $L_{l}$. In particular, this is the case in models with a single compact dimension. For a massive field, this kind of behavior in a locally AdS bulk is in contrast to that for a locally flat background geometry, where the decay is exponential, like $e^{-m L_{l}}$. Note that the leading terms in both the cases (5.16) and (5.17) do not depend on the location of the brane and coincide with the corresponding terms in the brane-free geometry investigated in [23]. In Fig. 6, the current density is plotted versus $L / z_{0}$ for fixed values of $z / z_{0}=1.05$ (left panel) and $z / z_{0}=1.2$ (right panel) and for the values of the phase $\tilde{\alpha}=2 \pi / 3$ (a), $\tilde{\alpha}=\pi / 2$ (b), $\tilde{\alpha}=\pi / 3$ (c).

\section{FERMIONIC CURRENTS IN $Z_{2}$-SYMMETRIC BRANEWORLDS}

In this section, we consider the applications of the results given above in Randall-Sundrum type braneworlds [6] with a single brane. For the model described in [34], the background geometry contains two copies of the R region, $y<0$ and $y>0$, and the line element is given by (2.1) with the warp factor replaced by $e^{-2|y| / a}$. The regions $y<0$ and $y>0$ are related by the $Z_{2}$-symmetry identification $y \leftrightarrow$ $-y$ and $y=0$ is the location of a positive tension brane. In the original setup, the standard model fields are localized on the brane and there is a single extra dimension $y$ $(D=4)$. Most scenarios motivated from string theories predict the presence of additional bulk fields and also small extra compact dimensions originating from 10D string backgrounds. Here we consider a $(D+1)$-dimensional generalization of the 1-brane model with an arbitrary number of toroidally compactified spatial dimensions. It will be assumed that the brane is located at $y=y_{0}$.

The boundary conditions for the fermionic field $\psi(x)$ on the brane are dictated by the $Z_{2}$-symmetry of the model. As a consequence of that symmetry we expect that $\psi\left(x^{i}, y_{0}-y\right)=M \psi\left(x^{i}, y-y_{0}\right)$ with a $N \times N$ matrix $M$. From the invariance of the action under the $Z_{2}$ identification, the following conditions are obtained (see also [12] for the case $D=4$ )

$$
\left\{\gamma^{(0)}, M\right\}=0, \quad\left[\gamma^{(0)} \gamma^{(b)}, M\right]=0, \quad\left\{\gamma^{(0)} \gamma^{(D)}, M\right\}=0,
$$

with $b=1,2, \ldots, D-1$. Now it can be easily checked that these conditions are satisfied by the choice $M=\zeta \gamma^{(D)}$. From the condition $M^{2}=1$, one gets $\zeta^{2}=-1$ and, hence, $\zeta= \pm i$. By taking into account the expression for $\gamma^{(D)}$ from (3.1) we find

$$
M=\mp s \operatorname{diag}(1,-1) .
$$

This matrix is unitary. With the choice (6.2) one gets the same boundary conditions on the brane for $s=1$ and $s=-1$. For the mode functions (3.3), we obtain the boundary condition $Z_{m a+1 / 2}\left(\lambda z_{0}\right)=0$ for the upper sign in (6.2) and the boundary condition $Z_{m a-1 / 2}\left(\lambda z_{0}\right)=0$ for the lower sign.

First let us consider the choice of the upper sign in (6.2). In this case, the boundary condition imposed on the function $Z_{m a+1 / 2}(\lambda z)$ coincides with that for the bag boundary condition discussed in Sec. V. Consequently, the expressions for the current density in the $Z_{2}$-symmetric braneworld model coincide with those obtained in Sec. V with an additional factor $1 / 2$. The appearance of the latter is related to the fact that in braneworlds the integration over $y$ in the normalization condition (3.6) goes over two copies of the $\mathrm{R}$ region and, as a consequence, the normalization coefficient for the modes is halved.

For the case with the lower sign in (6.2), from the condition $Z_{m a-1 / 2}\left(\lambda z_{0}\right)=0$ it follows that the mode functions are given by

$$
\begin{aligned}
\psi_{\beta}^{(+)}= & C_{\beta}^{(+)} z^{\frac{D+1}{2}} e^{i \mathbf{k} \mathbf{x}-i \omega t} \\
& \times\left(\begin{array}{c}
\frac{\mathbf{k} \chi \chi_{0}^{\dagger}+i \lambda-\omega}{\omega} g_{m a-1 / 2, m a+s / 2}\left(\lambda z_{0}, \lambda z\right) w^{(\sigma)} \\
i \chi_{0}^{\dagger} \frac{\mathbf{k} \chi \chi_{0}^{\dagger}+i \lambda+\omega}{\omega} g_{m a-1 / 2, m a-s / 2}\left(\lambda z_{0}, \lambda z\right) w^{(\sigma)}
\end{array}\right), \\
\psi_{\beta}^{(-)}= & C_{\beta}^{(-)} z^{\frac{D+1}{2}} e^{i \mathbf{k} \mathbf{x}+i \omega t} \\
& \times\left(\begin{array}{c}
i \chi_{0} \frac{\mathbf{k} \chi^{\dagger} \chi_{0}-i \lambda+\omega}{\omega} g_{m a-1 / 2, m a+s / 2}\left(\lambda z_{0}, \lambda z\right) w^{(\sigma)} \\
\frac{\mathbf{k} \chi^{\dagger} \chi_{0}-i \lambda-\omega}{\omega} g_{m a-1 / 2, m a-s / 2}\left(\lambda z_{0}, \lambda z\right) w^{(\sigma)}
\end{array}\right)
\end{aligned}
$$

with

$$
\left|C_{\beta}^{( \pm)}\right|^{2}=\lambda \frac{\left[J_{m a-1 / 2}^{2}\left(\lambda z_{0}\right)+Y_{m a-1 / 2}^{2}\left(\lambda z_{0}\right)\right]^{-1}}{8(2 \pi)^{p} V_{q} a^{D}}
$$

The reason for the appearance of an additional factor $1 / 2$ in the normalization coefficients is the same as that for the case of the upper sign in (6.2). Substituting the mode functions into the formula (3.7), by transformations similar to that we have demonstrated in the case of the bag boundary condition, for the VEV of the current density we find

$$
\begin{aligned}
\left\langle j^{l}\right\rangle= & \frac{1}{2}\left\langle j^{l}\right\rangle_{0}-\frac{N e A_{p} z^{D+2}}{2 V_{q} a^{D+1}} \sum_{\mathbf{n}_{q}} k_{l} \int_{k_{(q)}}^{\infty} d u u\left(u^{2}-k_{(q)}^{2}\right)^{\frac{p-1}{2}} \\
& \times \frac{I_{m a-1 / 2}\left(u z_{0}\right)}{K_{m a-1 / 2}\left(u z_{0}\right)}\left[K_{m a+1 / 2}^{2}(u z)-K_{m a-1 / 2}^{2}(u z)\right],
\end{aligned}
$$

where $\left\langle j^{l}\right\rangle_{0}$ is given by (4.9). 
An alternative representation, obtained by applying the summation formula (4.21), is given by

$$
\begin{aligned}
\left\langle j^{l}\right\rangle= & -\frac{N e a^{-D-1} z^{D+2}}{2(2 \pi)^{p / 2+1} V_{q} L_{l}^{p}} \sum_{r=1}^{\infty} \frac{\sin \left(r \tilde{\alpha}_{l}\right)}{r^{p+1}} \sum_{\mathbf{n}_{q-1}} \int_{0}^{\infty} d \lambda \lambda \\
& \times g_{p / 2+1}\left(r L_{l} \sqrt{\lambda^{2}+k_{(q-1)}^{2}}\right) \\
& \times \frac{\left.\sum_{j= \pm 1} g_{m a-1 / 2, m a+j / 2}^{2}\left(\lambda z_{0}, \lambda z\right)\right)}{J_{m a-1 / 2}^{2}\left(\lambda z_{0}\right)+Y_{m a-1 / 2}^{2}\left(\lambda z_{0}\right)} .
\end{aligned}
$$

Yet another representation is derived by rotating the integration contour in a way similar to that described in Sec. V. The corresponding formula is obtained from (5.12) with additional factor $1 / 2$ and by the replacement

$$
\frac{I_{m a+1 / 2}\left(\lambda z_{0}\right)}{K_{m a+1 / 2}\left(\lambda z_{0}\right)} \rightarrow-\frac{I_{m a-1 / 2}\left(\lambda z_{0}\right)}{K_{m a-1 / 2}\left(\lambda z_{0}\right)} .
$$

The asymptotic behavior of the current density in various limiting cases is investigated in a way similar to that in the previous section.

An important difference, compared with the case of the boundary condition corresponding to the upper sign in (6.2), is the behavior of the brane-induced VEV in (6.5) for the mass range $m a<1 / 2$ in the limit when the location of the brane tends to the AdS boundary, $z_{0} \rightarrow 0$. In this range of masses, we use the relation [32]

$$
\frac{I_{m a-1 / 2}\left(u z_{0}\right)}{K_{m a-1 / 2}\left(u z_{0}\right)}=\frac{2}{\pi} \cos (\pi m a)+\frac{I_{1 / 2-m a}\left(u z_{0}\right)}{K_{1 / 2-m a}\left(u z_{0}\right)} .
$$

The part of the current density corresponding to the last term in (6.8) vanishes in the limit $z_{0} \rightarrow 0$ like $z_{0}^{1-2 m a}$, whereas the part with the first term in the right-hand side of (6.8) does not depend on $z_{0}$. Hence, the brane-induced contribution in (6.5) (the second term on the right) tends to a finite limiting value:

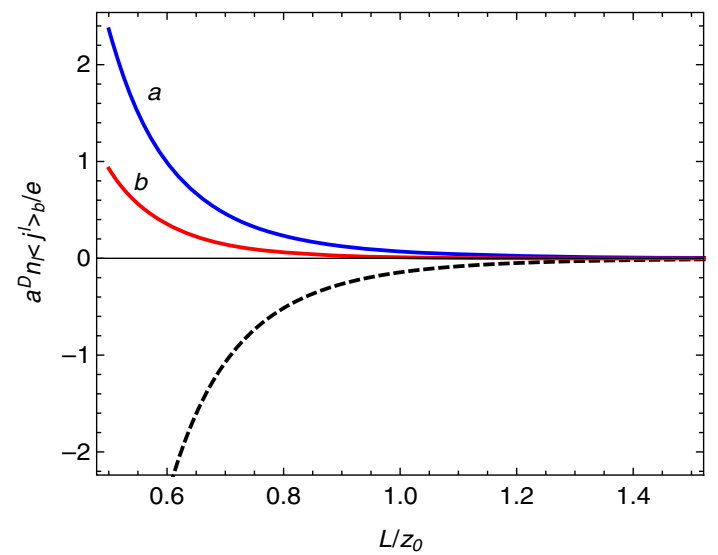

$$
\begin{aligned}
\lim _{z_{0} \rightarrow 0}\left\langle j^{l}\right\rangle_{b}= & -\frac{N e A_{p} z^{D+2}}{\pi V_{q} a^{D+1}} \cos (\pi m a) \sum_{\mathbf{n}_{q}} k_{l} \int_{k_{(q)}}^{\infty} d u u \\
& \times\left(u^{2}-k_{(q)}^{2}\right)^{\frac{p-1}{2}}\left[K_{m a+1 / 2}^{2}(u z)-K_{m a-1 / 2}^{2}(u z)\right],
\end{aligned}
$$

with $m a<1 / 2$. The limiting value of the total current density in this range of masses is obtained from (6.6) taking the limit $z_{0} \rightarrow 0$ :

$$
\begin{aligned}
\lim _{z_{0} \rightarrow 0}\left\langle j^{l}\right\rangle= & -\frac{N e a^{-D-1} z^{D+2}}{2(2 \pi)^{p / 2+1} V_{q} L_{l}^{p}} \sum_{r=1}^{\infty} \frac{\sin \left(r \tilde{\alpha}_{l}\right)}{r^{p+1}} \sum_{\mathbf{n}_{q-1}} \int_{0}^{\infty} d \lambda \lambda \\
& \times g_{p / 2+1}\left(r L_{l} \sqrt{\lambda^{2}+k_{(q-1)}^{2}}\right) \\
& \times\left[J_{-1 / 2-m a}^{2}(\lambda z)+J_{1 / 2-m a}^{2}(\lambda z)\right] .
\end{aligned}
$$

For a massless field, the brane-induced contribution vanishes and in (6.10) the brane-free contribution survives only.

We have taken the parameter $\zeta$ in the expression for the matrix $M$ the same for the representations $s=1$ and $s=-1$. We could take $\zeta= \pm i$ and in this case the matrix $M=\mp$ $\operatorname{diag}(1,-1)$ is the same for both the representations. However, with this choice, the boundary conditions on the brane are different for the representations $s=1$ and $s=-1$ : $Z_{m a+s / 2}\left(\lambda z_{0}\right)=0$ for the upper sign and $Z_{m a-s / 2}\left(\lambda z_{0}\right)=0$ for the lower one.

In the Randall-Sundrum-type models, our Universe is realized as a brane and from the point of view of the interpretation of the results described above by an observer living on the brane it is of interest to consider the current density on the brane. For a field with the boundary condition corresponding to the upper sign in (6.2), the VEV is given by (5.10) with the additional factor $1 / 2$. For the boundary condition with the lower sign in (6.2), the current density on the brane is directly obtained from (6.6) with $z=z_{0}$ :

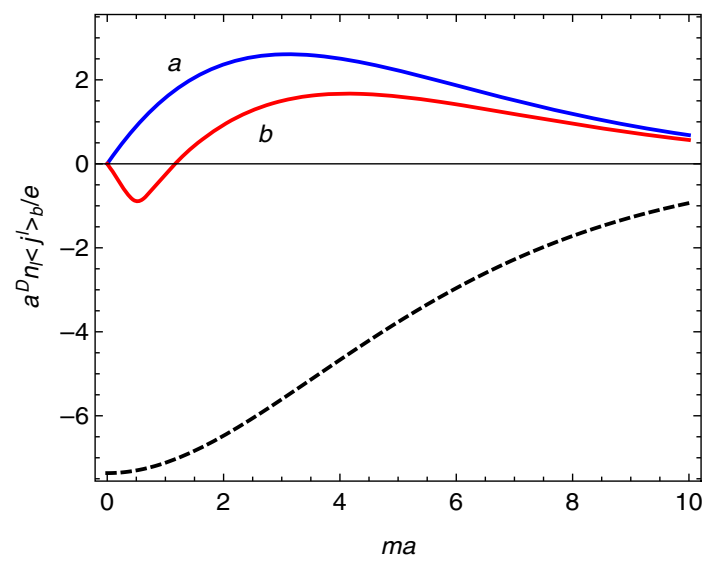

FIG. 7. The brane-induced current density on the brane in $Z_{2}$-symmetric $D=5$ models with a single compact dimension. The curves a and $\mathrm{b}$ are for the upper and lower signs in (6.2). For the left panel, we have taken $\tilde{\alpha}_{l}=\pi / 3$ and $m a=2$ and for the right panel $\tilde{\alpha}_{l}=\pi / 3$ and $L / z_{0}=0.5$. 


$$
\begin{aligned}
\left\langle j^{l}\right\rangle= & -\frac{8 N e a^{-D-1} z_{0}^{D}}{(2 \pi)^{p / 2+3} V_{q} L_{l}^{p}} \sum_{r=1}^{\infty} \frac{\sin \left(r \tilde{\alpha}_{l}\right)}{r^{p+1}} \\
& \times \sum_{\mathbf{n}_{q-1}} \int_{0}^{\infty} \frac{d u}{u} \frac{g_{p / 2+1}\left(r L_{l} \sqrt{u^{2} / z_{0}^{2}+k_{(q-1)}^{2}}\right)}{J_{m a-1 / 2}^{2}(u)+Y_{m a-1 / 2}^{2}(u)} .
\end{aligned}
$$

In Fig. 7, we have plotted the brane-induced contributions in the current density on the brane for the simplest generalization of the Randall-Sundrum type model with a single extra compact dimension of the length $L$. In this model, $D=5, p=3, q=1$. The curves a and b correspond to the upper and lower signs in (6.2). The dashed curve presents the current density at $z=z_{0}$ in the absence of the brane. The left panel presents the dependence of the current density on the ratio $L / z_{0}$ for fixed $\tilde{\alpha}_{l}=\pi / 3$ and $m a=2$. On the right panel, the brane-induced current density is plotted versus the mass (in units of $1 / a$ ) for the same value of $\tilde{\alpha}_{l}$ and for $L / z_{0}=0.5$. Comparing the current density on the brane (left panel in Fig. 7) and outside the brane (Fig. 6) as a function of the length $L$, we see the completely different behavior for small values of $L$. On the brane, the brane-induced current density increases with decreasing $L$, whereas outside the brane, for $L<\left|z-z_{0}\right|$, it is suppressed by the factor $e^{-2\left|z-z_{0}\right|\left|\tilde{\alpha}_{l}\right| / L}$.

\section{PARITY AND TIME-REVERSAL SYMMETRIC ODD-DIMENSIONAL MODELS}

We have considered the fermionic current density for a field realizing the irreducible representation of the Clifford algebra. In odd-dimensional spacetimes, the mass term $m \bar{\psi} \psi$ in the Lagrangian density is not invariant under the charge conjugation $(C)$ in $D=4 n$, under the parity $(P)$ transformation in $D=4 n, 4 n+2$, and under the time reversal $(T)$ in $D=4 n+2$ with $n=0,1,2, \ldots$ (of course, $C P T$ invariance holds in all dimensions, see, e.g., Ref. [35]). For even $D$, the matrix $\gamma^{(D)}$ is expressed in terms of the other Dirac matrices as $\gamma^{(D)}= \pm \gamma$ in $D=4 n$ and $\gamma^{(D)}= \pm i \gamma$ in $D=4 n+2$, with $\gamma=\prod_{i=0}^{D-1} \gamma^{(i)}$. The upper and lower signs correspond to the two inequivalent irreducible representations of the Clifford algebra. One can construct models invariant under the $P, C$, and $T$ transformations combining two $\mathrm{N}$-component fermionic fields $\psi_{(s)}, s= \pm 1$, realizing these representations. These fields correspond to the cases $s=1$ and $s=-1$ in the discussion above. In the gauge $A_{\mu}=0$, the Lagrangian density in the model is given by

$$
\mathcal{L}=\sum_{s= \pm 1} \bar{\psi}_{(s)}\left[i \gamma_{(s)}^{\mu}\left(\partial_{\mu}+\Gamma_{\mu}^{(s)}\right)-m\right] \psi_{(s)},
$$

where $\gamma_{(s)}^{\mu}=\left(\gamma^{0}, \gamma^{1}, \ldots \gamma^{D-1}, \gamma_{(s)}^{D}\right)$ and $\gamma_{(s)}^{D}=(z / a) \gamma^{(D)}$ with $\gamma^{(D)}$ from (3.1). By suitable transformations of the fields (see, e.g., Ref. [35]) we can see that (7.1) is invariant under the $C$-, $P$ - and $T$-transformations. Now the VEV of the total current density is the sum of the VEVs coming from the separate fields: $\left\langle J^{\mu}\right\rangle=\sum_{s= \pm 1}\left\langle j_{(s)}^{\mu}\right\rangle$. As it has been shown above, if the phases in the quasiperiodicity conditions for the fields $\psi_{(s)}$ are the same then the corresponding current densities coincide and the total current density is given by the expressions in Secs. IV and V with an additional factor 2. However, one can have situations where the phases along compact dimensions are different for separate irreducible representations. An example will be discussed below.

We can present the Lagrangian density (7.1) in terms of a single $2 N$-component spinor field $\Psi=\left(\psi_{(+1)}, \psi_{(-1)}\right)^{T}$ as

$$
\mathcal{L}=\bar{\Psi}\left[i \gamma^{(2 N) \mu}\left(\partial_{\mu}+\Gamma_{\mu}^{(2 N)}\right)-m\right] \Psi,
$$

with $2 N \times 2 N$ Dirac matrices

$$
\gamma^{(2 N) \mu}=\left(\begin{array}{cc}
\gamma_{(+1)}^{\mu} & 0 \\
0 & \gamma_{(-1)}^{\mu}
\end{array}\right)
$$

and the related spin connection $\Gamma_{\mu}^{(2 N)}$. An equivalent representation is obtained in terms of the fields $\psi_{(s)}^{\prime}=$ $[1+s+(1-s) \gamma] \psi_{(s)} / 2$. The combined Lagrangian density is rewritten as $\mathcal{L}=\sum_{s= \pm 1} \bar{\psi}_{(s)}^{\prime}\left[i \gamma^{\mu}\left(\partial_{\mu}+\Gamma_{\mu}\right)-s m\right] \psi_{(s)}^{\prime}$, where now $\gamma^{\mu}=\gamma_{(+1)}^{\mu}$ and $\Gamma_{\mu}$ is the related spin connection. Now the Lagrangian densities for separate fields are the same except the sign of the mass term. We can also write the Lagrnagian density in terms of $2 N$-component spinor $\Psi^{\prime}=$ $\left(\psi_{(+1)}^{\prime}, \psi_{(-1)}^{\prime}\right)^{T}$ as $\mathcal{L}=\bar{\Psi}^{\prime}\left[i \gamma^{\prime(2 N) \mu}\left(\partial_{\mu}+\Gamma_{\mu}^{\prime(2 N)}\right)-m\right] \Psi^{\prime}$ with $\gamma^{\prime(2 N) \mu}=\sigma_{\mathrm{P} 3} \otimes \gamma^{\mu}, \Gamma_{\mu}^{\prime(2 N)}=I \otimes \Gamma_{\mu}$.

In view of wide applications of the $D=2$ fermionic model in planar condensed matter systems (so called Dirac materials, including graphene, topological insulators and Weyl semimetals), here we consider in detail this special case. The low-energy excitations of the electronic subsystem in these materials are described by the Dirac equation where the velocity of light is replaced by a Fermi velocity $v_{F}$. In graphene, the Dirac equation is written for a 4-component spinor field. The upper and lower two-component fields of the latter correspond to inequivalent points, $\mathbf{K}_{+}$and $\mathbf{K}_{-}$, of the Brillouin zone, whereas their separate components correspond to the amplitude of the electron wave function on the triangular sublattices $A$ and $B$ of the graphene honeycomb lattice (for reviews see, e.g., [36]). Hence, in the Dirac model describing graphene the spinor field is presented as $\Psi_{S}=\left(\psi_{+, A S}, \psi_{+, B S}, \psi_{-, A S}, \psi_{-, B S}\right)^{T}$, where $S= \pm 1$ corresponds to spin degrees of freedom. The parameter $s= \pm 1$ in our previous discussion corresponds to the points $\mathbf{K}_{ \pm}$. In the special case of $D=2$ bulk geometry with $\Gamma_{0}^{(4)}=0$, and for the external electromagnetic field with the potential $A^{\mu}=(\varphi=0, \mathbf{A})$, in standard units the Lagrangian density reads 


$$
\begin{aligned}
\mathcal{L}= & \sum_{S= \pm 1} \bar{\Psi}_{S}\left[i \hbar \gamma^{(4) 0} \partial_{t}+i \hbar v_{F} \gamma^{(4) l}\left(\partial_{l}+\Gamma_{l}^{(4)}\right.\right. \\
& \left.\left.-i e A_{l} / \hbar c\right)-\Delta\right] \Psi_{S},
\end{aligned}
$$

where the summation goes over $l=1,2$ and $A_{l}$ is the $l$ th component of the spatial vector $\mathbf{A}$ (for electrons $e=-|e|$ ). In (7.4), the energy gap $\Delta$ is included which is related to the Dirac mass by the formula $\Delta=m v_{F}^{2}$. The corresponding Compton wavelength is given by $a_{\mathrm{C}}=\hbar v_{F} / \Delta$. Note that, depending on the gap generation mechanism (see, for instance, [36]) the mass term may have more complicated matrix structure. For a given $S$, the Lagrangian density in (7.4) is an analog of (7.2) for $D=2$.

Though the topology of the background space for the theory described by (7.4) is trivial in a planar graphene sheet, nontrivial topology can be realized by graphene made structures like carbon nanotubes and nanoloops with the topologies $R^{1} \times S^{1}$ and $S^{1} \times S^{1}$, respectively. The corresponding ground state currents induced by the threading magnetic flux have been investigated in [17] (neglecting the curvature effects for nanoloops). The periodicity conditions on the fields along compact dimensions of these structures are determined by the chirality of the tube. In the case of metallic nanotubes and in the absence of threading magnetic flux, the periodic boundary condition $(\alpha=0)$ is realized for both the fields $\psi_{( \pm 1)}=\left(\psi_{ \pm, A S}, \psi_{ \pm, B S}\right)$. In semiconducting nanotubes for the phases corresponding to the fields $\psi_{( \pm 1)}$, one has $\alpha=$ $\pm 2 \pi / 3$ and they have opposite signs for spinors corresponding to the points $\mathbf{K}_{+}$and $\mathbf{K}_{-}$of the Brillouin zone (see, e.g., [37]). As a result of that, the contributions in the current density coming from these points cancel each other and, in the absence of the magnetic flux, the total current density is zero for both metallic and semiconducting nanotubes.

The topology in the problem at hand is that realized in carbon nanotubes, however the tube here is curved (the effects of curvature in graphene made structures have been discussed in $[38,39])$. The $\mathrm{R}$ region of corresponding spatial geometry with an edge, embedded in three-dimensional Euclidean space $R^{3}$, is depicted in Fig. 8. The magnetic flux enclosed by the compact dimension is shown separately. A similar type of spatial geometry has been considered in [39], though, the geometry of spacetime differs from that we consider here. Curved graphene tubes with wormhole geometry were discussed in [40].

For $D=2$, the spatial geometry corresponding to (2.1) is described by the line element $d l^{2}=d y^{2}+L^{2} e^{-2 y / a} d \varphi^{2}$, where $x^{1}=L \varphi, \varphi \in[0,2 \pi]$. This corresponds to the line element on Beltrami pseudosphere with Gaussian curvature $-1 / a^{2}$. It is known that the complete Beltrami pseudosphere cannot be embedded in $R^{3}$ with Cartesian coordinates $(X, Y, Z)$. However, this can be done for a part of Beltrami pseudosphere corresponding to $y \geq y_{0} \equiv$ $a \ln (L / a)$. For the conformal coordinate $z$, one has $z \geq L$. The corresponding parametrization is given by (see also the discussion in Ref. [39])

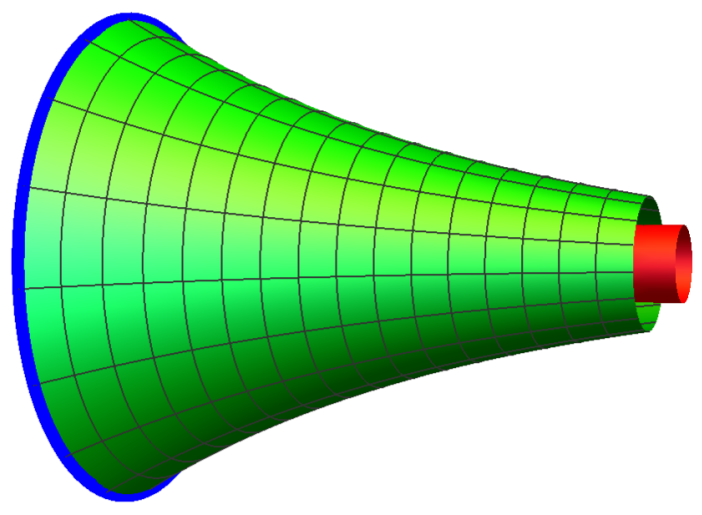

FIG. 8. The spatial geometry of deformed $D=2$ tube with an edge, threaded by a magnetic flux and embedded in threedimensional Euclidean space.

$X=f(y), \quad Y=L e^{-y / a} \cos \varphi, \quad Z=L e^{-y / a} \sin \varphi$,

where $f(y)=\int_{y_{0}}^{y} d u \sqrt{1-L^{2} e^{-2 u / a} / a^{2}}$. The edge of the embedded surface (7.5) is a circle in the plane $X=0$ with radius $a$. Having embedded a part of Beltrami pseudosphere, it is a trivial task to embed the corresponding subspace of $(2+1)$-dimensional spacetime geometry with the line element $d s^{2}=d t^{2}-d y^{2}-L^{2} e^{-2 y / a} d \varphi^{2}$ in $(3+1)$-dimensional Minkowski spacetime with the coordinates $(T, X, Y, Z)$ (see, for instance, [39]). However, the embedding is not trivial for $(2+1)$-dimensional geometry under consideration (or a subspace of that) with the line element $d s^{2}=e^{-2 y / a}\left(d t^{2}-L^{2} d \varphi^{2}\right)-d y^{2}$ having $g_{00} \neq 1$. Note that the nontrivial 00-component of the effective metric tensor for electrons in graphene can be generated by various mechanisms. They include external fields, the deformations of graphene lattice (strains), and the local variations in the Fermi velocity (see, for instance, Ref. [41] and references therein). It is of interest to specify the mechanism that can mimic the gravitational field we have considered. This requires a separate consideration and will be addressed in our future work.

In the presence of the magnetic flux, the total current is obtained by summing the contributions from the fields $\psi_{(+1)}$ and $\psi_{(-1)}$, given in the previous sections (specified to $D=2$, $q=1$ ), with the opposite signs of the phases. As an example, we present here the current density in the region $z \geq z_{0}$, for a given spin degree of freedom $S$, obtained from (5.9):

$$
\begin{aligned}
\left\langle J^{1}\right\rangle= & \frac{2 e v_{F} z^{4}}{\pi a^{3} z_{0}^{3}} \sum_{r=1}^{\infty} \cos (r \alpha) \sin \left(2 \pi r \Phi / \Phi_{0}\right) \int_{0}^{\infty} d u u^{2} \\
& \times K_{1}\left(r u L / z_{0}\right) \frac{g_{\nu, \nu}^{2}\left(u, u z / z_{0}\right)+g_{\nu, \nu-1}^{2}\left(u, u z / z_{0}\right)}{J_{\nu}^{2}(u)+Y_{\nu}^{2}(u)},
\end{aligned}
$$

where $\alpha$ is the phase for the field $\psi_{(+1)}, \nu=a / a_{\mathrm{C}}+1 / 2$ and we have written the mass in terms of the Compton 


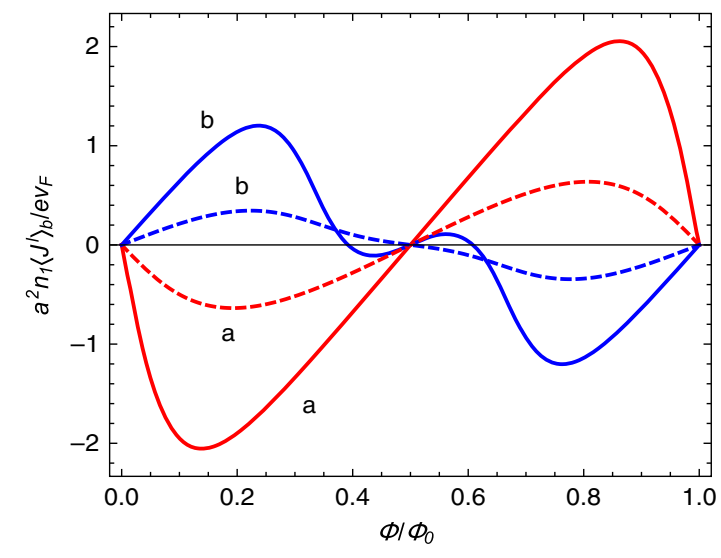

FIG. 9. The edge-induced current density as a function of the magnetic flux in $D=2$ metallic (a) and semiconducting (b) tubes for $a / a_{\mathrm{C}}=2, z / z_{0}=1.05, z / L=4$ (full curves) and $z / L=2$ (dashed curves).

wavelength. The appearance of $v_{F}$ in the coefficient of (7.6) is related to fact that the spatial components of the current density for a given $S$, corresponding to the Lagrangian (7.4), are defined as $J^{l}=e v_{F} \bar{\Psi}_{S} \gamma^{(4) l} \Psi_{S}$. Introducing in (7.6) a new integration variable $\lambda=u / z_{0}$ and taking the limit $z_{0} \rightarrow 0$, we obtain the current density $\left\langle J^{1}\right\rangle_{0}$ in the edge-free geometry with $0 \leq z<\infty$. In this limit, the integral is expressed as a derivative with respect to $L$ of the integral

$\int_{0}^{\infty} d \lambda \lambda K_{0}(n \lambda L)\left[J_{\nu}^{2}(\lambda z)+J_{\nu-1}^{2}(\lambda z)\right]=\frac{1}{n L z}\left(\frac{w-1}{w+1}\right)^{a / a_{\mathrm{C}}}$,

with $w=\sqrt{4 z^{2} /(n L)^{2}+1}$. It can be checked that the corresponding expression is reduced to that given in [23]. In Fig. 9, we display the edge-induced contribution in (7.6), $\left\langle J^{1}\right\rangle_{b}=\left\langle J^{1}\right\rangle-\left\langle J^{1}\right\rangle_{0}$, as a function of the magnetic flux (in units of the flux quantum) for metallic ( $\alpha=0$, curves a) and semiconducting ( $\alpha=2 \pi / 3$, curves b) quasiperiodicity conditions. The full and dashed curves correspond to $z / L=4$ and $z / L=2$, respectively. The graphs are plotted for $a / a_{\mathrm{C}}=2$ and $z / z_{0}=1.05$.

The fermionic current density induced by a magnetic flux in planar graphene rings with circular edges has been recently investigated in [42]. The fermionic field was confined in the circular ring by the bag boundary conditions on the edges. In the corresponding problem, in addition to the azimuthal current, a nonzero VEV of the charge density appears. Note that persistent currents of a similar physical origin, appearing in normal metal rings, have been detected in [43].

\section{CONCLUSION}

We have investigated the properties of the vacuum state for a charged fermionic field constrained by two sorts of boundary conditions. The first one is related to the topology of the background spacetime, being locally AdS with an arbitrary number of toroidally compactified spatial dimensions. Along those dimensions the field obeys quasiperiodicity conditions (2.5) with general phases. The toroidal compactification does not change the local geometry and, because of the high symmetry of the AdS spacetime, the problem we consider is exactly solvable. The second sort of boundary condition is imposed by the presence of a brane parallel to the AdS boundary. We consider the bag boundary condition that is the most popular condition used for the confinement of fermions in a variety of situations (an example is the MIT bag model for hadrons). In addition to the background gravitational field, we also assume the presence of a constant Abelian gauge field. The gauge transformation excluding the latter from the field equation, leads to the shift of the phases in the quasiperiodicity conditions for the new field operator. This shift can be formally interpreted in terms of a magnetic flux enclosed by the compat dimension. Yet another kind of boundary condition should be imposed on normalizable irregular modes at the timelike boundary of the AdS spacetime. Here we consider a special case of allowed boundary conditions corresponding to the bag boundary condition on a hypersurface close to the AdS boundary with the subsequent limiting transition to the AdS boundary.

Because of the global nature of the vacuum in quantum field theory, the expectation values of local physical observables are sensitive to the boundary conditions on the field. As such a local observable we consider the fermionic current density. The corresponding VEVs for the charge density and for the components of the current along noncomapct dimensions vanish. The brane divides the space into two regions with different properties of the vacuum and we consider the VEVs for the components of the current along compact dimensions in these regions separately.

In the region between the brane and AdS boundary, the fermionic modes are presented as (4.1) and the eigenvalues of the quantum number $\lambda$ are quantized by the boundary condition on the brane. In order to extract from the VEV of the current density the contribution induced by the brane, we have applied to the series over these eigenvalues the summation formula (4.6). For the component along the $l$ th compact dimension, this contribution is given by (4.15). It is an odd periodic function of the phase $\tilde{\alpha}_{l}$ and an even periodic function of the remaining phases $\tilde{\alpha}_{i}, i \neq l$, with the period equal to $2 \pi$. For a massless field, the result on the locally AdS bulk is conformally related to the corresponding result on a locally Minkwoski bulk in the region between two parallel planar boundaries. The latter are conformal images of the AdS boundary and of the brane. For a massive field, we have also checked the limiting transition to the corresponding result in the problem with a single boundary on a locally Minkowski spacetime. On the AdS boundary, both the brane-free and brane-induced contributions to the current density tend to zero as 
$z^{D+2 m a+1}$. An important feature that distinguishes the VEV of the current density from the VEV of the energymomentum tensor is the finiteness of the former on the brane. For the investigation of the near-brane asymptotic, we have provided an alternative representation (4.23). The limiting value of the current density on the brane is directly obtained from that representation and is given by (4.28). The simple relation (4.29) takes place between the limiting value and the integrated current density in the $\mathrm{L}$ region. For $z \gg L_{i}$ and at distances from the brane corresponding to $z_{0}-z \gg L_{i}$ the brane-induced contribution behaves as (4.31) and it is mainly localized near the brane in the region $z_{0}-z \lesssim L_{i}$. For a fixed observation point, when the brane is close to the AdS horizon, the brane-induced current density is suppressed by the factor $e^{-2 k_{(q)}^{(0)} z_{0}}$, with $k_{(q)}^{(0)}$ defined by (4.32) for $\left|\tilde{\alpha}_{i}\right|<\pi$. If the length of the $l$ th compact dimension is much smaller than the lengths of the remaining dimensions, the leading term in the expansion of the component $\left\langle j^{l}\right\rangle_{b}$ coincides with the current density in the model with a single compact dimension $x^{l}$ when the remaining compact dimensions are decompactified. If, in addition, one has $L_{l} \ll z_{0}-z$, then the brane-induced current density decays like $e^{-2\left(z_{0}-z\right)\left|\tilde{\alpha}_{l}\right| / L_{l}}$ (see (4.39)). This feature is seen in Fig. 3. In the opposite limit of large values for $L_{l}$, the brane-induced current density along the corresponding dimension behaves as (4.40) and it is suppressed by the factor $\exp \left[-L_{l} \sqrt{\lambda_{1}^{2} / z_{0}^{2}+k_{(q-1)}^{(0) 2}}\right]$.

In the region between the brane and the AdS horizon, the fermionic mode functions are given by (5.3) and the eigenvalues for $\lambda$ are continuous. The brane-induced contribution to the VEV of the current density in this region is presented in the form (5.8). An alternative expression for the total current in the R region is given by (5.9), with the limiting value on the brane expressed as (5.10). For a massless field, the problem is conformally related to the corresponding problem on a locally Minkowskian spacetime with a single boundary and the brane-induced contribution vanishes in the $\mathrm{R}$ region. In the case of a massive field and at large distances from the brane $\left(z \gg z_{0}, L_{i}\right.$, points near the AdS horizon), that contribution behaves as (5.14) and is suppressed by the factor $e^{-2 z k_{(q)}^{(0)}}$. In this region, the total current density is dominated by the brane-free part. For a fixed observation point, when the brane location tends to the AdS boundary, $z_{0} \rightarrow 0$, the brane-induced current density tends to zero as $z_{0}^{2 m a+1}$. If the length $L_{l}$ is much smaller than the lengths of the remaining compact dimensions and also $L_{l} \ll z-z_{0}$, similar to the case of the $\mathrm{L}$ region, the brane-induced current decays as $e^{-2\left(z-z_{0}\right)\left|\tilde{\alpha}_{l}\right| / L_{l}}$. In the opposite limit of large values for $L_{l}$, the asymptotic behavior of the current is essentially different depending on the phases in the periodicity conditions along the remaining compact dimensions. If at least one of the phases $\tilde{\alpha}_{i}$, $\left|\tilde{\alpha}_{i}\right|<\pi, i \neq l$, is different from zero, the leading term in the asymptotic expansion is given by (5.16) with an exponential suppression like $e^{-L_{l} k_{(q-1)}^{(0)}}$. In the case $\tilde{\alpha}_{i}=0$, the decay of the current density as a function of $L_{l}$ is power law for both massive and massless fields.

In $Z_{2}$-symmetric braneworlds of the Randall-Sundrum type, the geometry is composed by two copies of the $\mathrm{R}$ region related by the $Z_{2}$-symmetry identification. Depending on the transformation of the field under the $Z_{2}$-reflection two types of the boundary conditions on the brane are obtained. The first one corresponds to the bag boundary condition, considered in Sec. V. The current density in this case coincides with that investigated in Sec. V with an additional factor $1 / 2$, related to the presence of two copies of the $\mathrm{R}$ region. For the second boundary condition, the eigenvalues of the quantum number $\lambda$ are the zeros of the function $Z_{m a-1 / 2}\left(\lambda z_{0}\right)$, where $z_{0}$ is the location of the brane. The VEV of the current density in this case is given by (6.5) or, alternatively, by (6.6). Now, in the range of the mass $m a<1 / 2$, the brane-induced current density does not vanish in the limit when the brane tends to the AdS boundary. The limiting values for the brane-induced and total currents are given by (6.9) and (6.10). In the RandallSundrum models, the observers reside on the brane and the current density measured by them is presented as (6.11). The corresponding dependencies on the length of compact dimension and on the mass for two types of boundary conditions are exemplified in Fig. 7.

In odd-dimensional spacetimes, the mass term for a field realizing the irreducible representation of the Clifford algebra breaks the invariance with respect two of the $C$-, $P$ - and $T$-transformations. Models invariant under these transformations are constructed by combining the fields realizing two inequivalent representations of the Clifford algebra. We have shown that, if the phases in the quasiperiodicity conditions are the same for these fields, they give the same contribution to the total current density in this kind of models. From the point of view of applications of fermionic models in condensed matter physics, an important special case corresponds to threedimensional spacetime. An example of physical realization of those models is graphene. The corresponding low-energy excitations of the electronic subsystem are described by the Lagrangian density (7.4). The background topology in this model is nontrivial for carbon nanotubes and nanoloops (cylindrical and toroidal topologies, respectively). The phase for the fermionic field along compact dimension of the nanotube depends on the chirality of the tube. For deformed tubes with $z$-dependent radius, the resulting current is given by (7.6), where $\alpha=0$ and $\alpha=2 \pi / 3$ for metallic and semiconducting tubes, respectively. As a consequence of the cancellation of the contributions from the points $\mathbf{K}_{+}$and $\mathbf{K}_{-}$of the Brillouin zone, the current vanishes in the absence of the magnetic flux $\Phi$ threading the tube. 


\section{ACKNOWLEDGMENTS}

A. A. S. gratefully acknowledges the hospitality of the INFN, Laboratori Nazionali di Frascati (Frascati, Italy), where a part of this work was done. V. V. V. acknowledges support through a De Sitter cosmology fellowship.

[1] S. J. Avis, C. J. Isham, and D. Storey, Phys. Rev. D 18, 3565 (1978); P. Breitenlohner and D. Z. Freedman, Phys. Lett. B 115, 197 (1982).

[2] C. J. Isham, Proc. R. Soc. A 362, 383 (1978); 364, 591 (1978); J. S. Dowker and R. Banach, J. Phys. A 11, 2255 (1978); R. Banach and J. S. Dowker, J. Phys. A 12, 2527 (1979).

[3] X.-L. Qi and S.-C. Zhang, Rev. Mod. Phys. 83, 1057 (2011); A. Furusaki, N. Nagaosa, K. Nomura, S. Ryu, and T. Takayanagi, C.R. Phys. 14, 871 (2013); A. Tanaka and S. Takayoshi, Sci. Technol. Adv. Mater. 16, 014404 (2015).

[4] C. G. Callan, Jr. and F. Wilczek, Nucl. Phys. B340, 366 (1990).

[5] O. Aharony, S. S. Gubser, J. Maldacena, H. Ooguri, and Y. Oz, Phys. Rep. 323, 183 (2000); H. Năstase, Introduction to AdS/CFT Correspondence (Cambridge University Press, Cambridge, 2015); M. Ammon and J. Erdmenger, Gauge/Gravity Duality: Foundations and Applications (Cambridge University Press, Cambridge, England, 2015).

[6] R. Maartens and K. Koyama, Living Rev. Relativity 13, 5 (2010).

[7] A.S. T. Pires, AdS/CFT Correspondence in Condensed Matter (Morgan \& Claypool Publishers, California, 2014); J. Zaanen, Y.-W. Sun, Y. Liu, and K. Schalm, Holographic Duality in Condensed Matter Physics (Cambridge University Press, Cambridge, 2015); R.-G. Cai, L. Li, L.-F. Li, and R.-Q. Yang, Sci. China Phys. Mech. Astron. 58, 060401 (2015); E. Kiritsis and L. Li, J. High Energy Phys. 01 (2016) 147.

[8] V. M. Mostepanenko and N. N. Trunov, The Casimir Effect and Its Applications (Oxford University Press, New York, 1997); K. A. Milton, The Casimir Effect: Physical Manifestation of Zero-Point Energy (World Scientific, Singapore, 2002); M. Bordag, G. L. Klimchitskaya, U. Mohideen, and V. M. Mostepanenko, Advances in the Casimir Effect (Oxford University Press, Oxford, 2009); Casimir Physics, edited by D. Dalvit, P. Milonni, D. Roberts, and F. da Rosa, Lecture Notes in Physics Vol. 834 (Springer-Verlag, Berlin, 2011).

[9] L. P. Teo, J. High Energy Phys. 10 (2010) 019.

[10] E. Elizalde, S. D. Odintsov, and A. A. Saharian, Phys. Rev. D 87, 084003 (2013).

[11] A. Flachi, I. G. Moss, and D. J. Toms, Phys. Lett. B 518, 153 (2001).

[12] A. Flaci, I. G. Moss, and D. J. Toms, Phys. Rev. D 64, 105029 (2001).

[13] L. P. Teo, Int. J. Mod. Phys. A 28, 1350158 (2013).

[14] V.E. Ambruş and E. Winstanley, Phys. Lett. B 749, 597 (2015); Classical Quantum Gravity 34, 145010 (2017).
[15] A. Flachi, J. Garriga, O. Pujolàs, and T. Tanaka, J. High Energy Phys. 08 (2003) 053; A. Flachi and O. Pujolàs, Phys. Rev. D 68, 025023 (2003); A. A. Saharian, Phys. Rev. D 73, 044012 (2006); 73, 064019 (2006); 74, 124009 (2006); E. Elizalde, M. Minamitsuji, and W. Naylor, Phys. Rev. D 75, 064032 (2007); R. Linares, H. A. Morales-Técotl, and O. Pedraza, Phys. Rev. D 77, 066012 (2008); M. Frank, N. Saad, and I. Turan, Phys. Rev. D 78, 055014 (2008).

[16] E. R. Bezerra de Mello and A. A. Saharian, Phys. Rev. D 87, 045015 (2013).

[17] S. Bellucci, A. A. Saharian, and V. M. Bardeghyan, Phys. Rev. D 82, 065011 (2010).

[18] S. Bellucci, E. R. Bezerra de Mello, and A. A. Saharian, Phys. Rev. D 89, 085002 (2014).

[19] S. Bellucci and A. A. Saharian, Phys. Rev. D 87, 025005 (2013).

[20] S. Bellucci, A. A. Saharian, and N. A. Saharyan, Eur. Phys. J. C 75, 378 (2015).

[21] S. Bellucci, A. A. Saharian, and H. A. Nersisyan, Phys. Rev. D 88, 024028 (2013).

[22] E. R. Bezerra de Mello, A. A. Saharian, and V. Vardanyan, Phys. Lett. B 741, 155 (2015).

[23] S. Bellucci, A. A. Saharian, and V. Vardanyan, Phys. Rev. D 96, 065025 (2017).

[24] S. Bellucci, A. A. Saharian, and V. Vardanyan, J. High Energy Phys. 11 (2015) 092.

[25] S. Bellucci, A. A. Saharian, and V. Vardanyan, Phys. Rev. D 93, 084011 (2016).

[26] E. Elizalde, Ten Physical Applications of Spectral Zeta Functions (Springer-Verlag, Berlin, 1995); F. C. Khanna, A. P. C. Malbouisson, J. M. C. Malbouisson, and A.E. Santana, Phys. Rep. 539, 135 (2014).

[27] L.E. Parker and D. J. Toms, Quantum Field Theory in Curved Spacetime: Quantized Fields and Gravity (Cambridge University Press, Cambridge, 2009).

[28] P. Breitenlohner and D. Z. Freedman, Ann. Phys. (N.Y.) 144, 249 (1982); L. Mezincescu and P. K. Townsend, Ann. Phys. (N.Y.) 160, 406 (1985); S. Bellucci, Phys. Rev. D 35, 1296 (1987); 36, 1127 (1987); M. Henningson and K. Sfetsos, Phys. Lett. B 431, 63 (1998); W. Mueck and K. S. Viswanathan, Phys. Rev. D 58, 106006 (1998).

[29] A. J. Amsel and D. Marolf, Classical Quantum Gravity 26, 025010 (2009).

[30] O. Aharony, D. Marolf, and M. Rangamani, J. High Energy Phys. 02 (2011) 041.

[31] A. A. Saharian, Izvestiia Akademii nauk Armianskoi SSR Matematika 22, 166 (1987) [Sov. J. Contemp. Math. Anal. 22, 70 (1987)]; arXiv:0708.1187.

[32] Handbook of Mathematical Functions, edited by M. Abramowitz and I. A. Stegun (Dover, New York, 1972). 
[33] A. P. Prudnikov, Yu. A. Brychkov, and O. I. Marichev, Integrals and Series (Gordon and Breach, New York, 1986), Vol. 2.

[34] L. Randall and R. Sundrum, Phys. Rev. Lett. 83, 3370 (1999); 83, 4690 (1999).

[35] K. Shimizu, Prog. Theor. Phys. 74, 610 (1985).

[36] V. P. Gusynin, S. G. Sharapov, and J. P. Carbotte, Int. J. Mod. Phys. B 21, 4611 (2007); A. H. Castro Neto, F. Guinea, N. M. R. Peres, K. S. Novoselov, and A. K. Geim, Rev. Mod. Phys. 81, 109 (2009).

[37] T. Ando, J. Phys. Soc. Jpn. 74, 777 (2005).

[38] D. V. Kolesnikov and V. A. Osipov, Phys. Part. Nucl. 40, 502 (2009); M. A. H. Vozmediano, M. I. Katsnelson, and F. Guinea, Phys. Rep. 496, 109 (2010).

[39] A. Iorio and G. Lambiase, Phys. Rev. D 90, 025006 (2014).
[40] J. González and J. Herrero, Nucl. Phys. B825, 426 (2010); V. Atanasov and A. Saxena, J. Phys. Condens. Matter 23, 175301 (2011); R. Pincak and J. Smotlacha, Eur. Phys. J. B 86, 480 (2013); A. Sepehri, R. Pincak, K. Bamba, S. Capozziello, and E. N. Saridakis, Int. J. Mod. Phys. D 26, 1750094 (2017).

[41] G. E. Volovik and M. A. Zubkov, Ann. Phys. (Amsterdam) 356, 255 (2015); B. Amorim et al., Phys. Rep. 617, 1 (2016).

[42] S. Bellucci, A. A. Saharian, and A. Kh. Grigoryan, Phys. Rev. D 94, 105007 (2016).

[43] H. Bluhm, N. Koshnick, J. Bert, M. Huber, and K. Moler, Phys. Rev. Lett. 102, 136802 (2009); A. C. Bleszynski-Jayich, W. E. Shanks, B. Peaudecerf, E. Ginossar, F. von Oppen, L. Glazman, and J. G. E. Harris, Science 326, 272 (2009). 